\title{
Rheology signature of flocculated silica suspensions
}

\author{
Jennifer Fusier, ${ }^{1}$ Julie Goyon, ${ }^{1}$ Xavier Chateau, ${ }^{1, a)}$ and Fabrice Toussaint ${ }^{2}$ \\ ${ }^{1}$ Université Paris-Est, Laboratoire Navier (UMR 8205), CNRS, Ecole des Ponts ParisTech, IFSTTAR, \\ F-77455 Marne-la-Vallée, France \\ ${ }^{2}$ LafargeHolcim, 38070 Saint Quentin Fallavier, France
}

(Received 19 May 2017; final revision received 22 January 2018; published 25 April 2018)

\begin{abstract}
We experimentally study the behavior of suspensions of silica particles in aqueous solution. Despite many studies on these materials, the dependence of the overall rheological properties of the suspension on particle size, solid volume fraction, ionic strength, and strain history remains debated. In this paper, we manage to manufacture materials and develop procedures that allow us (i) to approach this problem in the best possible way and (ii) to check that the results obtained with well controlled systems (monodisperse silica spherical particles) also apply to less controlled suspensions (crushed silica particles). We find that the elastic modulus-particle size and yield stress-particle size relationships follow power laws that disagree with classical models from the literature. We also show that elastic modulus versus yields stress data fall on a single master curve when rescaled by particle size, whatever are solid volume fraction, resting time, and ionic strength. This suggests that the rescaled elastic modulus can play the role of a parameter in a structural kinetics model of the behavior of thixotropic suspensions. Furthermore confocal observations of the system provided evidence that the evolution of the overall properties of the material with resting time cannot be ascribed to changes in the particle network. (C) 2018 The Society of Rheology. https://doi.org/10.1122/1.5031897
\end{abstract}

\section{INTRODUCTION}

Paste systems, which consist of colloidal particles (up to several micrometers) suspended in a fluid, are present in many industries, such as civil engineering or the food industry. For example, fresh cement paste (i.e., cement paste before setting and hardening) is a suspension of irregularly shaped particles with a typical size range from $1 \mu \mathrm{m}$ to about $10 \mu \mathrm{m}$. In the concrete industry, the placement of the material is an important operation which largely determines the quality of the final product. The placement of the paste in formworks or its spreading on solid surfaces relies on the rheology of the material. Proving rheology is a key parameter of concrete technology [1].

As it is well known, colloidal suspensions' response to a loading does not depend only on the stress or strain applied at the concerned time, but also depends on the stress-strain history experienced by the material [2-5]. This behavior finds its origin at the particle scale: When suspended in water, colloidal particles organize spatially as a result of particle-particle interaction (van der Waals, steric, etc.), fluidparticle interaction (electrostatic, Brownian motion, etc.), or others [6]. Rheology of colloidal suspension is mainly controlled by particle interactions, and a lot of work has been devoted to study the link between interactions at the particle scale and the overall behavior of the suspension [2,5,7-11]. Thanks to these works, consensus has emerged to attribute the intricate overall properties of attractive colloidal particle

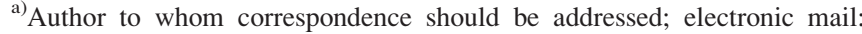
xavier.chateau@enpc.fr
}

suspensions to the changes in the particle microstructure induced by flow.

If neighboring particles attract each other and if the suspension is at rest for a while, particles will cluster together to form flocs. As flocs are porous and behave as rigid particles when slowly sheared by the suspending fluid, flocculation increases the apparent viscosity of the suspension. Moreover, if colloidal aggregates span over the sample, the viscosity diverges and the material has a yield stress. Conversely, it is possible to break aggregates and then to redisperse the colloidal particles over the suspending fluid by strongly shearing the suspension. It is well known that many thixotropic materials such as ketchup, bentonite, or cement paste (among others) exhibit these flow-induced and time changes of apparent viscosity [2-5]. As all these interactions depend upon the particles' size and shape as well as on the suspending fluid properties the same dependencies exist for the overall suspension properties.

A better understanding of the parameters that control the rheology of attractive colloidal suspensions is essential to make progress in this field. A better comprehension of the link between phenomena occurring at the particle scale and the overall behavior of the material will surely enhance the design of new materials or help in developing materials with improved or more controlled properties. For this purpose, many recent research works have focused on studying the rheological properties of mineral suspensions [9,12-14] and more specifically silica suspensions [8,15-22]. It has been observed that for most of the studied systems, the yield stress depends on the solid volume fraction through a power-law $[9,14,15,23]$. The same tendency has been observed regarding the elastic shear modulus' dependence on the solid 
volume fraction by [24]. In all these works, it has also been observed that the interaction forces magnitude strongly impacts the material properties. Furthermore, it was unambiguously observed in several of these studies that adding salt enhances flocculation of mineral suspensions. On the other hand, it seems that the time dependency of yield stress and shear modulus have received little attention [17,21].

Particle properties, especially size, also greatly interfere in the rheological response. It has been observed for many systems that the yield stress is inversely proportional to the square of particle's size $[9,25]$ even it is difficult to draw a definitive conclusion due to the small range of particle's size used in most of the experimental studies. Even if some models agree with these experimental results [10], a consensus on the modeling of this trend has not yet emerged since other models predict an inverse dependence of the yield stress on the particle's size $[2,26,27]$. Flatt and Bowen discuss the influence of rugosity on particle's size-yield stress dependence in the framework of the Yodel model [10]. They reach to the conclusion that yield stress is inversely proportional to the square of particles size for rough particles whereas an inverse dependence is predicted for both particles. To our best knowledge, experimental validation of this result remains to be made.

Studies have also been carried out to model and link the rheological properties to the fractal dimension of suspensions $[5,28,29]$. However, this concept is of limited applicability at a volume fraction above 0.2 .

Numerical simulations of colloidal suspensions allow to study the influence of particle interactions on the rheological properties of colloidal suspensions. Numerical simulations are attractive methods because they can incorporate all the desired information about the interparticle interaction, and then, allow to study how the suspension's rheological behavior depends on the model's ingredients. For example, the behavior of non-Brownian suspensions under shear flow conditions has been studied by Mari et al. [30] in the framework of Stokesian Dynamics model. They show that adding friction between solid particles to a model including hydrodynamic interactions and short-range repulsive potential is enough to reproduce shear thickening including its dependence on the solid volume fraction. Even if numeric simulations are versatile, enabling to model the behavior of colloidal aggregates under shear flow conditions $[31,32]$ or to reproduce the compressive consolidation behavior of strongly aggregated particle gels [33] they have to be validated by comparison to macroscopic experimental data due to the lack of experimental device for measuring the contact forces for any particle's size.

The problem of transitioning from the particle scale to the macroscopic scale in view of the prediction of overall behavior remains far from being fully understood.

In this article, we focus on the dependence of the yield stress and elastic modulus with respect to time, strength of particle interaction, solid volume fraction, and particle size. The behavior of real colloidal suspensions reveals many complex features that originate from the great variety of interactions and particle properties. We chose to work with model materials to reduce the number of parameters and phenomena involved in upscaling the suspensions properties. In this way, interactions and particles' properties should be more easily controlled, tuned, and described, which eases identification of the influence of microscopic parameters onto the overall properties. However, as a first step toward better understanding of real materials, we also perform experiments with irregularly shaped particles.

In Sec. II, we describe the materials employed and the experimental setup we used. Experimental results for the yield stress and elastic modulus are presented in Sec. III and compared to theoretical results from the literature in Sec. IV. Finally, Sec. V concludes and gives some perspectives about future work.

\section{MATERIAL AND METHODS}

To investigate the behavior of suspensions we use two types of silica particles suspended in an aqueous solution, respectively, monodisperse silica beads synthesized via the Stöber process and polydisperse crushed silica provided by Sibelco. First, a carefully controlled material is manufactured to reduce the number of parameters needed to describe its microscopic properties as much as possible. Then, as a first step toward the study of real materials, we also prepare suspensions with angular shaped particles with a broader size distribution. From now on, the synthesized silica beads will be called the model material and the crushed silica will be referred to as the realistic material even if it is clear that this material does not exhibit all the complexity and diversity of real materials.

The particle volume fraction of colloidal suspensions varies between approximately 0.3 and 0.4 . To control the degree of flocculation of suspensions, we only adjust the ionic strength through the addition of salt. We use divalent salt, $\mathrm{CaCl}_{2}$, which is commonly used in civil engineering materials, with an ionic strength $I$ varying from 0.05 to 0.3 . As we used the same salt in the whole study, we do not vary the valency of the type of ions. The ionic strength is defined as

$$
I=\frac{1}{2} \sum_{i} c_{i} z_{i}^{2}
$$

where $c_{i}$ is the ion concentration in $\mathrm{moll}^{-1}$ and $z_{i}$ the ion valency. Indeed the ionic strength controls the magnitude of interactions at play in the suspensions. Basically, the interactions involved in such systems are described by Derjaguin, Landau, Verwey, and Overbeek (DLVO) theory. DLVO [34,35] developed a theory explaining main trends of aqueous dispersion by combining two main interactions: van der Waals attraction [6,36] and electrostatic repulsive force [6]. The simplified expression for DLVO potential between two spheres of diameter $d_{p}$ in an asymmetric electrolyte separated by a distance $h$ is given by

$$
V_{\mathrm{DLVO}}=-\frac{A_{H} d_{p}}{24 h}+\pi d_{p} \varepsilon \varepsilon_{0} \Psi_{0}^{2} \exp ^{-\kappa h}
$$

with $A_{H}$ representing the Hamaker constant. Different values ranging from $10^{-21} \mathrm{~J}$ to $10^{-20} \mathrm{~J}$ can be found in the literature 
for the Hamaker constant of silica particles suspended in water [24]. $\kappa$ denotes the inverse of Debye length, $\varepsilon$ and $\varepsilon_{0}$ the relative permittivity of the medium and of the vacuum, respectively, and $\Psi_{0}$ is the surface potential.

It is worth noting that changing the salt concentration also induces a variation of the surface potential $\Psi_{0}$. To the best of our knowledge, a satisfactory model does not exist to predict $\Psi_{0}$ variations with respect to a change in the salt concentration. To overcome this difficulty, we experimentally measure $\Psi_{0}$ for the different salt concentrations values used in this study (see Sec. II A). From Eq. (2), it is readily seen that particles size influences both the van der Waals and electrostatic forces.

The particles also experience Brownian motion and gravitational force. To quantify their competitive effects, we introduce the Péclet number

$$
P e=\frac{\pi \Delta \rho g d_{p}^{4}}{12 k_{B} T}
$$

with $\Delta \rho$ as the difference between the particle density and the suspending medium density and $g$ as the gravity. For $T=293 \mathrm{~K}$ and silica particle with density $\rho \simeq 2000 \mathrm{~kg} \mathrm{~m}^{-3}$ and diameter $d_{p}=0.7 \mu \mathrm{m}$ suspended in water, we obtain $P e$ $\simeq 0.15$ whereas for the same particle with diameter $d_{p}=1.4 \mu \mathrm{m}$ we have $P e \simeq 2.5$. In our study, we are close to the limit between colloidal and noncolloidal systems, but it has to be noticed that this number does not take into account the interactions between particles in a flocculated state which prevent sedimentation with the apparition of yield stress.

\section{A. Materials}

\section{Model system: Silica beads}

The silica bead particles are produced by a Stöber synthesis [37]. The hydrolysis of silicon alkoxides in a basic medium leads to the precipitation of essentially monodisperse $\mathrm{SiO}_{2}$ particles through the chemical reactions

$$
\begin{gathered}
\equiv \mathrm{Si}-\mathrm{OR}+\mathrm{H}_{2} \mathrm{O} \stackrel{\mathrm{HO}}{\rightleftharpoons} \equiv \mathrm{Si}-\mathrm{OH}+\mathrm{ROH}, \\
\equiv \mathrm{Si}-\mathrm{OH}+\equiv \mathrm{Si}-\mathrm{OH} \rightleftharpoons \equiv \mathrm{Si}-\mathrm{O}-\mathrm{Si} \equiv+\mathrm{H}_{2} \mathrm{O}, \\
\equiv \mathrm{Si}-\mathrm{OH}+\equiv \mathrm{Si}-\mathrm{OR} \rightleftharpoons \equiv \mathrm{Si}-\mathrm{O}-\mathrm{Si} \equiv+\mathrm{ROH} .
\end{gathered}
$$

Many authors [38-41] have worked on improving the size control and reproducibility of this synthesis. Indeed the temperature, the type of alkoxides, solvents, and the rate of reagent addition are crucial to the final particles size as well as the monodispersity. We follow the protocol established by Kang et al. [42] with a few changes (details about the operating conditions are reported in Table I). Ethanol absolute $>99 \%(\mathrm{EtOH}$, Analar NORMAPUR) and ammonium hydroxide $28 \%\left(\mathrm{NH}_{4} \mathrm{OH}\right.$, AnalaR NORMAPUR) are mixed and tetraethyl orthosilicate for synthesis (TEOS, Merck) in ethanol is added dropwise with a syringe pump. The batch was stirred for $1 \mathrm{~h}$ after the addition of reagents. Then, particles are separated from the solvent by centrifugation and washed several times with ethanol 96\% (GPR Rectapur VWR) and deionized water to remove impurities
TABLE I. Operating conditions.

\begin{tabular}{lc}
\hline \hline & Quantities \\
\hline Ethanol absolute $(\mathrm{ml})$ & 490 (batch)/100 (dropwise) \\
$\mathrm{NH}_{4} \mathrm{OH}(\mathrm{ml})$ & 120 \\
TEOS $(\mathrm{ml})$ & 60 \\
Rate of adding $\left(\mathrm{ml} \mathrm{min}^{-1}\right)$ & 1.1 \\
\hline \hline
\end{tabular}

and traces of solvents. The particles are finally dried at $60{ }^{\circ} \mathrm{C}$ for several days.

Particles having diameters of $0.7,0.8,1.0$, and $1.4 \mu \mathrm{m}$ have been produced. The particles' size has been characterized using laser granulometry. A scanning electron microscope (SEM) image and a typical size distribution of particles are presented in Fig. 1. It is readily seen that particles are spherical and almost monodisperse. Standard deviation of the size distribution depicted in Fig. 1 is equal to $130 \mathrm{~nm}$, that is, $\simeq 10 \%$ of the particle's mean diameter.

Large amount of silica beads cannot be produced by Stöber synthesis; only about $20 \mathrm{~g}$ are produced per batch. Therefore, it was not possible to perform all the reproducibility tests we would have liked to do. We did not have this problem with crushed silica particles since they are available in large quantities.

\section{Crushed silica}

To check that the result obtained with silica spherical particles are of interest for real materials, we also studied suspensions of crushed silica. The quartz particles are also made of silica, but they are polydisperse and angularly shaped [Fig. 2(a)]. Quartz is extracted from a quarry and crushed. During this process, particles are washed and calcined up to $700{ }^{\circ} \mathrm{C}$, which remove most of the silanol groups $(\mathrm{SiOH})$ from the particles' surface by condensing water between the two silanol groups. To ensure that the quartz particles' surface properties are similar to that of silica beads, zeta potential measurements have been performed on calcined silica beads (see Sec. II A 4). Two average diameters are used: $2 \mu \mathrm{m}$ (C800, Sibelco) and $4 \mu \mathrm{m}$ (C600, Sibelco). The size distribution of these particles, measured by laser granulometry, is presented in Fig. 2(b). The particles are angular, polydisperse, and have a broad size distribution.

The properties of the different types of particles used in this study are summarized in Table II.

\section{Formulation protocol}

We observed that the rheological properties of our suspensions evolve with time and shear-stress history. Consequently, samples are prepared using a rigorously controlled procedure. In order to obtain homogeneous suspensions, the desired amount of dry powder is dispersed in water and mixed for several hours. Then, the solution is centrifuged to concentrate it at the desired volume fraction. The $p \mathrm{H}$ of the suspension is around 5.8 and varies up to 6.02 for the highest calcium chloride concentration. Therefore, the $p \mathrm{H}$ variation of the suspension by addition of $\mathrm{CaCl}_{2}$ can be neglected. The proper amount of salt is then added to the 


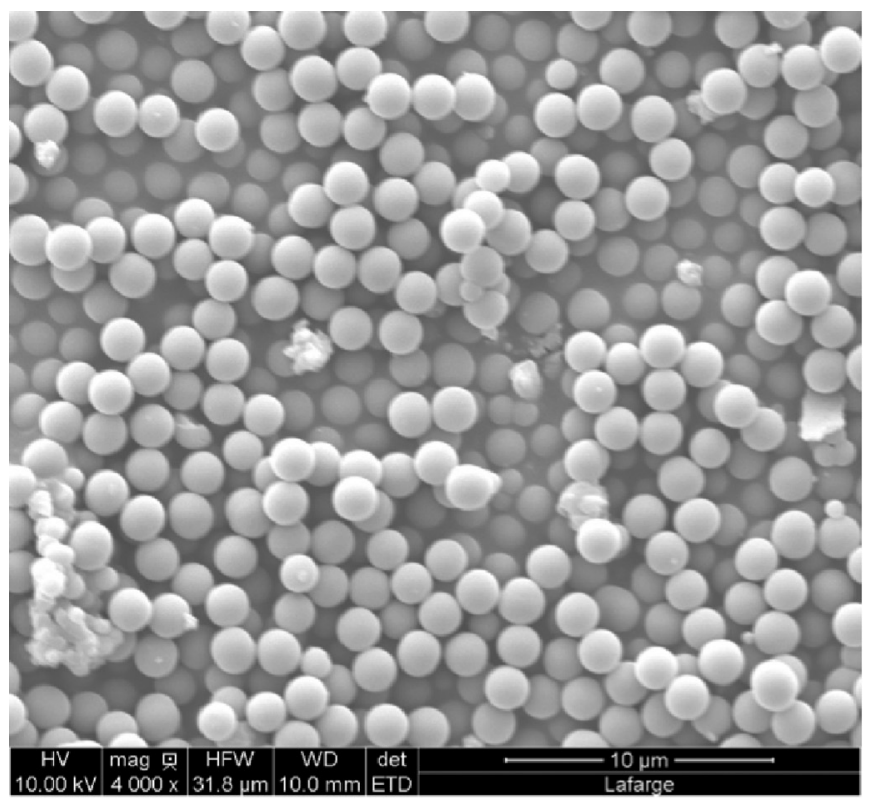

(a) SEM

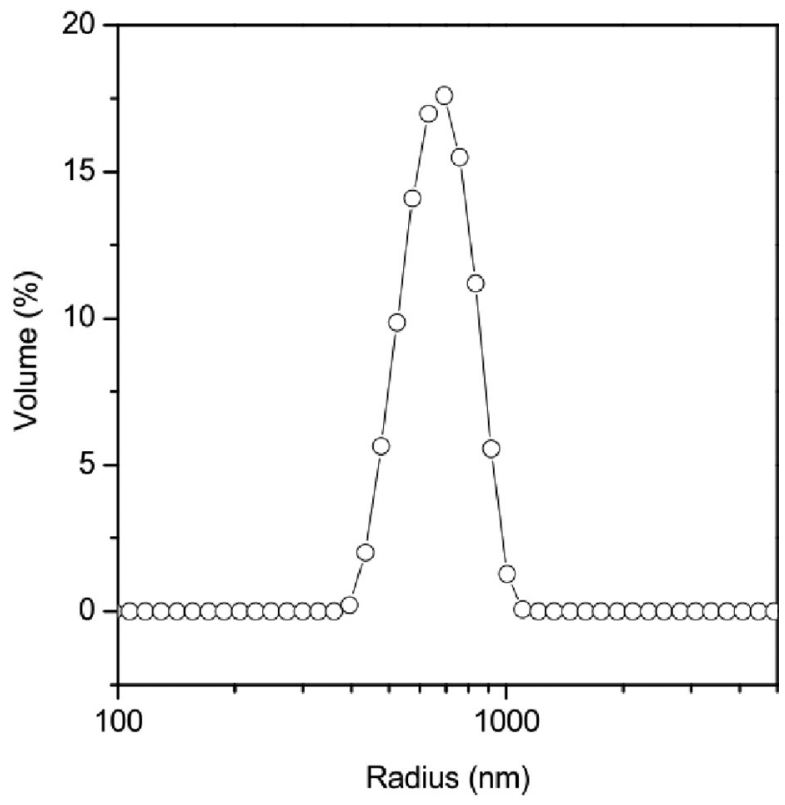

(b) DLS

FIG. 1. SEM picture (a) and size distribution measured by DLS (b) of $1.4 \mu \mathrm{m}$ silica beads.

suspension which is vigorously mixed until the system is homogeneous. Then, the rheometer cup is filled and sonicated for $2 \mathrm{~min}$ to deflocculate the material prior to placing it in the rheometer. The rheometry cycle starts 1 min after sonication. The exact volume fraction is measured by weighing the dry extract of a small amount of each sample dried at $60{ }^{\circ} \mathrm{C}$ in an oven.

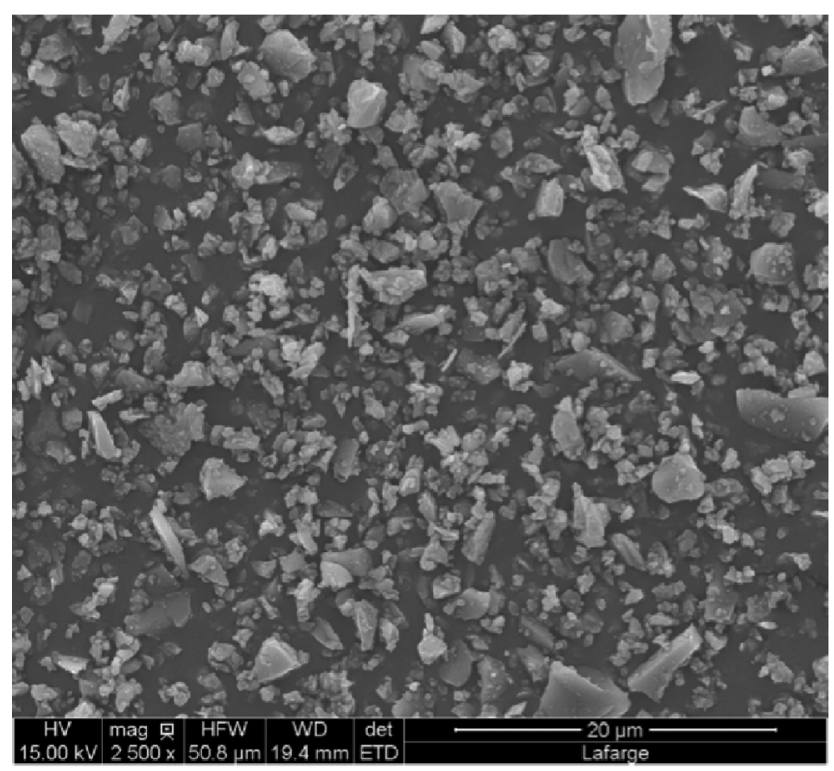

(a) SEM

\section{Zeta potential measurement}

Silica surfaces are known to be negatively charged in water [43]; the surface hydroxyl groups in contact with water form $\left(-\mathrm{O}^{-}\right)$groups, then the zeta potential measured is negative. Its magnitude varies when salt is added to the suspending fluid because the amplitude of the electrostatic repulsive potential is lowered. Indeed the only parameters impacted

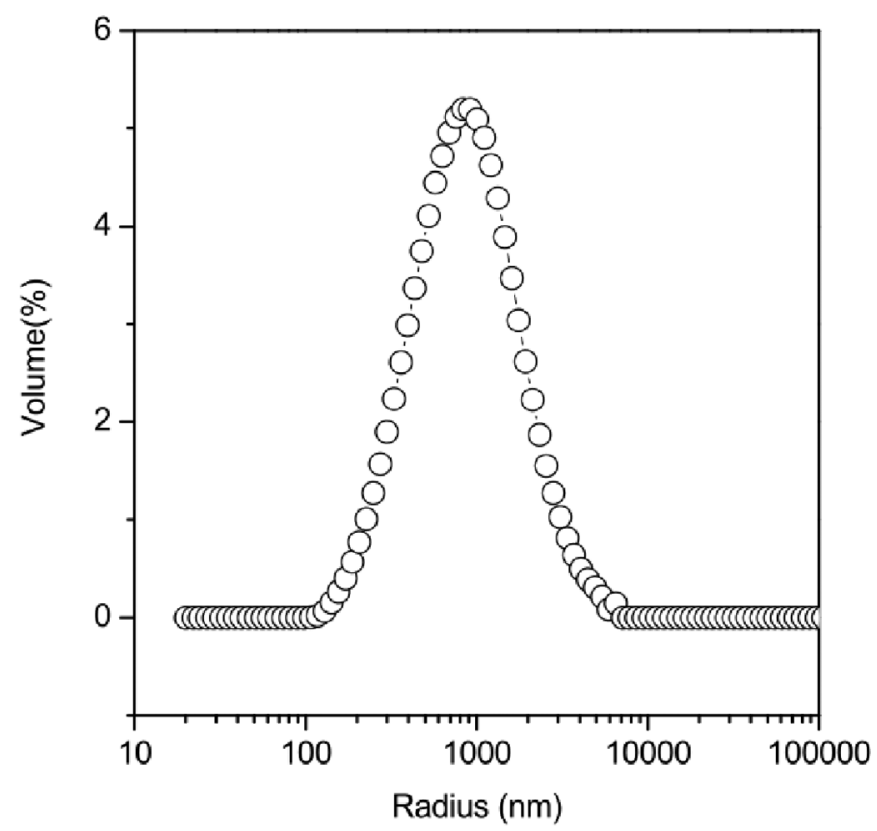

(b) Laser granulometry

FIG. 2. Two micrometer crushed silica particles observed with a SEM (a) and characterized by laser granulometry (b). 
TABLE II. Particles' properties.

\begin{tabular}{lcc}
\hline \hline Properties & Silica beads & Quartz \\
\hline Median diameter $(\mu \mathrm{m})$ & $0.7-1.4$ & $2 / 4$ \\
Size distribution & Monodisperse & Polydisperse \\
Specific surface area $\left(\mathrm{m}^{2} \mathrm{~g}^{-1}\right)$ & $7.1-2.7$ & $6.25 / 4.85$ \\
\hline \hline
\end{tabular}

are the ion concentration in Eq. (1) and the surface potential appearing in Eq. (2) for the electrostatic potential. It is extremely difficult to measure the potential at a particle surface due to the presence of adsorbed ions and solvent molecules near the solid surface, but it is possible to measure the potential at the shear plane between the particle and the suspending medium, thanks to electrophoretic mobility method [44].

The mobility of the particle submitted to an electric field is measured and related to its zeta potential by the Smoluchowski equation, which reads for $\kappa d_{p} \gg 1$,

$$
\mu=\frac{\Psi_{0} \varepsilon}{\eta},
$$

In Eq. (4), $\mu$ denotes the electrophoretic mobility of the particle and $\eta$ the medium viscosity. The zeta potential of suspensions with solid weight fraction of $0.005 \mathrm{wt}$. \% was measured using a zetasizer (Cad instrument) for ionic strengths varying from 0 to 0.1 . It was not possible with our instrument to measure zeta potentials for higher ionic strengths due to perturbing effects at high conductivity.

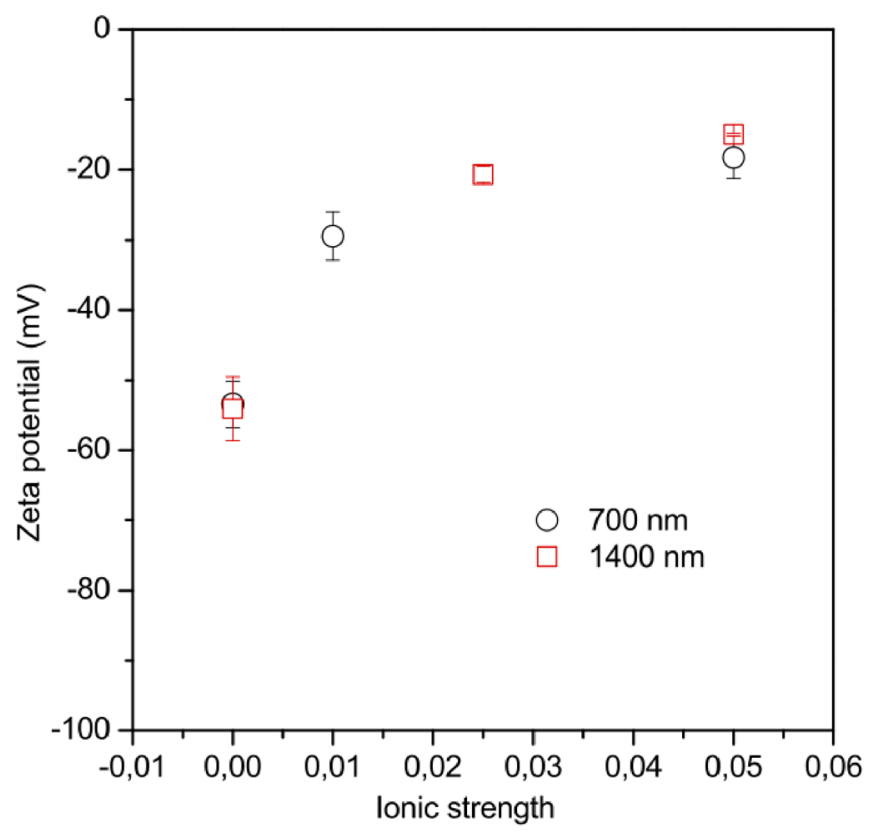

(a) Silica beads of $1.4 \mu \mathrm{m}$ (red square) and $0.7 \mu \mathrm{m}$ (black circle)
Nevertheless, Johnson et al. [45] showed by using electroacoustic techniques that the zeta potential of concentrated suspensions is close to that of dilute suspensions.

Many researchers assume that the magnitude of the zeta potential provides an indication about the stability of the colloidal system. For large (positive or negative) values of $\Psi_{0}$ particles tend to repulse each other and the suspension does not flocculate. On the contrary, if the particles have a low zeta potential, there is no force to prevent flocculation of the particles. There exists a particular value of the $p \mathrm{H}$, known as the isoelectric point, for which the majority of silanol groups are neutralized and the total net charge on the surface is zero. For silica, this $p \mathrm{H}$ is around $2-3$. We do not work in this domain, but at natural $p \mathrm{H}$ around 6 . One can readily see in Fig. 3(a) that adding salt decreases the magnitude of the zeta potential. Moreover, the zeta potential does not depend on particles' size.

Quartz particles are calcined during the industrial process. The only way we have to check that calcination does not change completely the surface properties is to compare the zeta potential before and after calcination (i.e., heated up $700^{\circ} \mathrm{C}$ ) of silica particles. Comparison was made for spherical silica beads because the Zeta potential measurement is possible only for spherical particles [Eq. (4) was obtained by assuming sphericity of the particles]. The ratio of the zeta potential of calcinated spherical particles to that of uncalcined spherical particles is depicted in Fig. 3(b) as a function of ionic strength. Calcination does not alter significantly the value of Zeta potential of the particles. Therefore, we believe

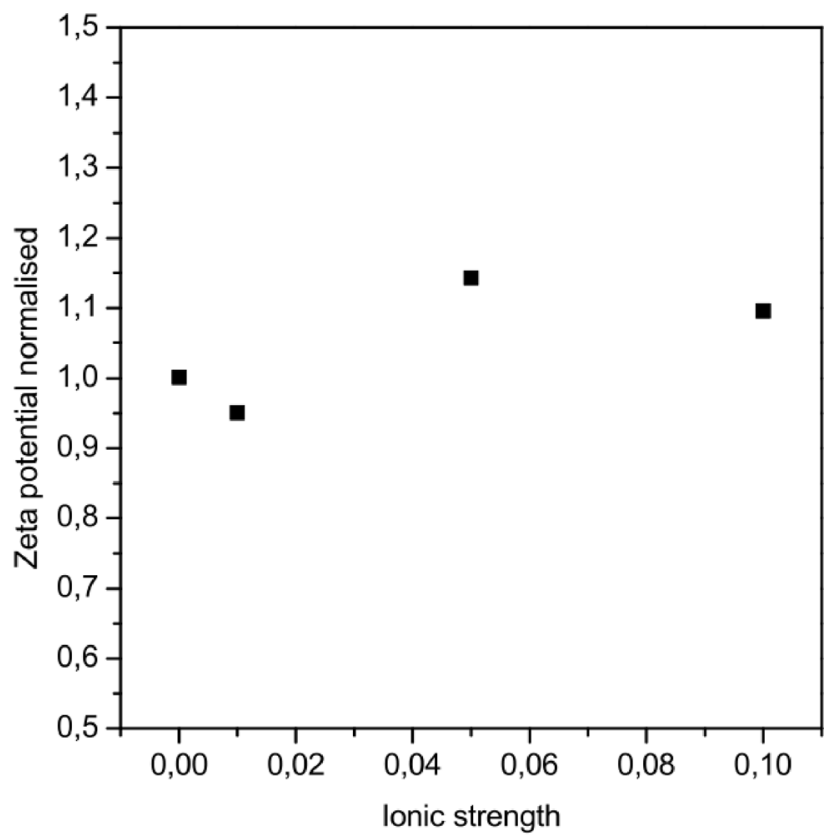

(b) $\Psi_{0}$ of calcined silica beads of $1.4 \mu \mathrm{m}$ normalised by $\Psi_{0}$ of uncalcined beads. 
that the difference in measured properties for silica beads and crushed particles should be attributed to the particle shape and polydispersity.

\section{Sedimentation}

As the density difference between silica particles and the aqueous solution is about $1 \mathrm{~kg} / \mathrm{m}^{3}$, we use the nonintrusive Proton Magnetic Resonance Imaging technique to investigate the sedimentation of the suspensions $[46,47]$. We study the time evolution of the horizontal section-averaged profile of the particle volume fraction. The material is poured in a cup with the diameter and height equal to $2 \mathrm{~cm}$ and then set to rest. The solid volume fraction profile is measured every $30 \mathrm{~min}$ for several hours. Results for a $1.4 \mu \mathrm{m}$ silica bead suspension at a solid volume fraction of 0.35 and an ionic strength of 0.15 are depicted in Fig. 4. It is readily seen that the solid volume fraction slightly increases at the bottom of the sample while a thin layer of water appears close to the free top surface. Overall, sedimentation is rather limited in the main part of the sample (the change in normalized solid volume fraction is less than $5 \%$ apart from the thin layer of water located at the top of the sample); the material remains globally homogeneous over $90 \mathrm{~min}$ and the solid volume fraction variation is less than $2 \%$ in the bottom of the sample and less than $1 \%$ in most of the sample. As rheometric measurements on each sample last approximately $1 \mathrm{~h}$, one might conclude that particle sedimentation does not perturb rheometric measurements.

\section{B. Rheometry measurements}

Since the suspensions are highly thixotrope, the rheometry measurements must be designed to account for the time dependence of rheological properties. We are mainly interested in the yield stress and in the elastic modulus of the

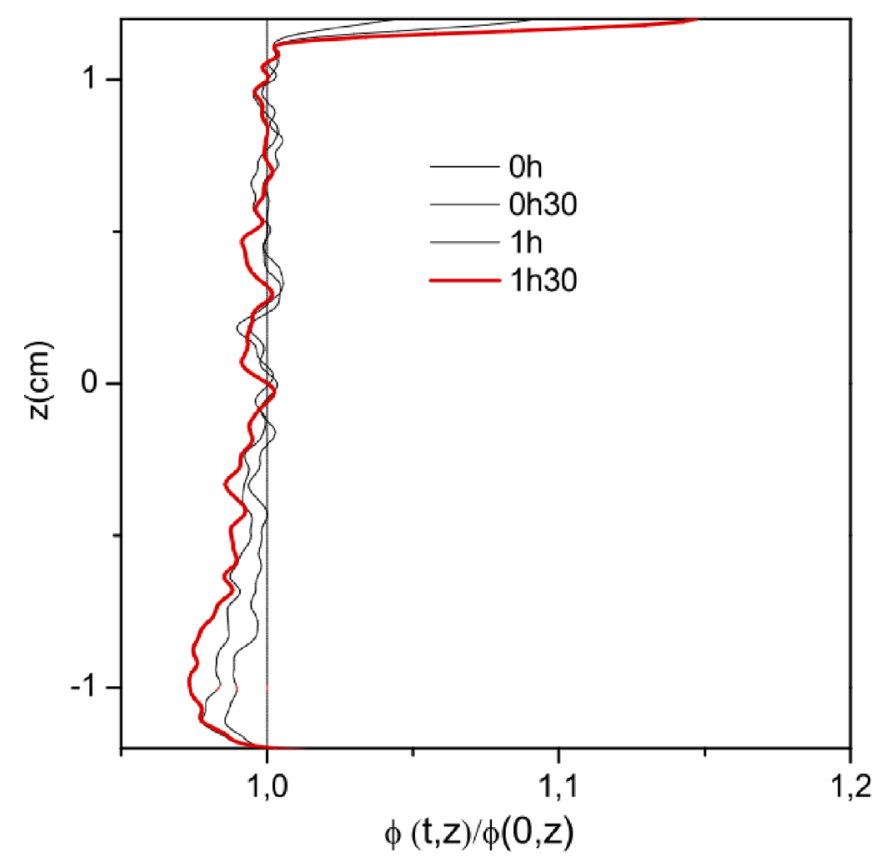

FIG. 4. Evolution of the normalized bead volume fraction profile measured in a suspension of $1.4 \mu \mathrm{m}$ silica bead suspension with homogeneous initial volume fraction $\phi_{v}=0.35$ and ionic strength $I=0.15$. suspensions. These two quantities complement each other since they provide additional information: Yield stress characterizes the macroscopic strength of the material, which is a quantity of primary interest for applications, whereas elastic modulus is strongly related to microstructure of suspensions and to the spatial organization of the suspended particles. We use a specific protocol to measure the evolution of these quantities with time. The experiments are performed within a thin gap Couette geometry (inner radius $\mathrm{R}_{i}=12.5 \mathrm{~mm}$, outer cylinder radius $\mathrm{R}_{e}=13.75 \mathrm{~mm}$, and height $\mathrm{H}=37.5 \mathrm{~mm}$ ) in a stress controlled rheometer (Kinexus Malvern) with rough surfaces to avoid slipping.

\section{Preshear}

To study the influence of resting time on the rheological properties of flocculated silica suspensions, several experiments are performed on the same sample. After pouring the material into the Couette cup, a strong initial shear is applied to "erase" the preparation and setting history. Then, a preshear at $200 \mathrm{~s}^{-1}$ is applied on the sample for $3 \mathrm{~min}$ before each measurement to start from a reference state of "rejuvenation." Then, a series of experiments is performed to measure the rheological properties of the sample.

It is not clear that the rejuvenation preshear fully deflocculates the material, i.e., that only single silica particles are suspended right after the rejuvenation process. Heiney et al. [17] observed that the flocculation of primary particles is irreversible on submicron sized silica particles. Nevertheless we checked that the rejuvenation procedure we used in this work allows us to reach a reproducible state and then to obtain satisfactory results. It is worth noting that our procedure ensures that the structural state of the colloidal suspension contained in the rheometer is homogeneous. Then, when the torque applied to the inner cylinder is increased from zero, the first material elements to flow are located near the inner cylinder because the shear stress is a decreasing function of the radius. As the elastic modulus is also measured on material samples that are homogeneously destructured, we do not face the classical problems resulting from heterogeneities of the structuration state when the sample experiences a complex strain-stress history. For greater certainty, we carefully checked that measured elastic modulus and yield stress do not depend on Couette gap device.

Figure 5 shows the evolution of the elastic modulus, measured using the procedure described below, as a function of time measured over three intervals $(5,10$, and $20 \mathrm{~min}$ ).

\section{Elastic modulus measurements method}

Each measurement begins just after the rejuvenation preshear was applied to the sample. As can be seen in Fig. 5, the evolution of the elastic modulus is similar for each of the three experiments.

Elastic modulus evolution during resting time is measured using a method well suited for thixotropic materials [48]. Small strain oscillations with a frequency equal to $1 \mathrm{~Hz}$ are applied to the sample during the resting period just after stopping the rejuvenation preshear. It is found that the measured elastic modulus does not depend on the frequency in 


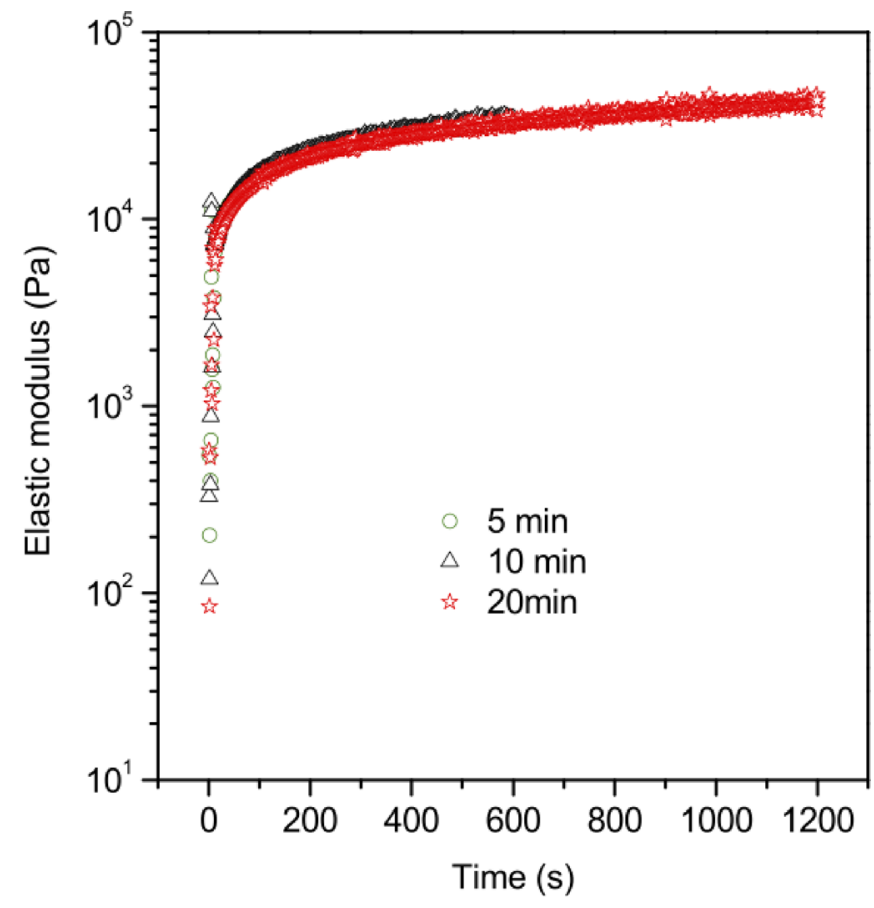

FIG. 5. Elastic modulus vs time with $\gamma=10^{-4}$ at $1 \mathrm{~Hz}$ frequency for $1.4 \mu \mathrm{m}$ suspension of silica beads at $\phi_{v}=0.35$ and $I=0.1$ after 5, 10, and $20 \mathrm{~min}$ of resting time (see Sec. II B 2 for the description of the elastic modulus measurement procedure).

the range $[0.5-2 \mathrm{~Hz}]$. Figure 6(a) shows that same evolution is obtained for the three frequency values: $0.5,1$, and $2 \mathrm{~Hz}$. The linear elastic domain (i.e., the domain in which strain depends linearly on stress) is identified by applying strain oscillations with increasing amplitude. The size of the linear domain, that is, to say, the small strain domain, evolves with resting time because of the thixotropy of suspensions,

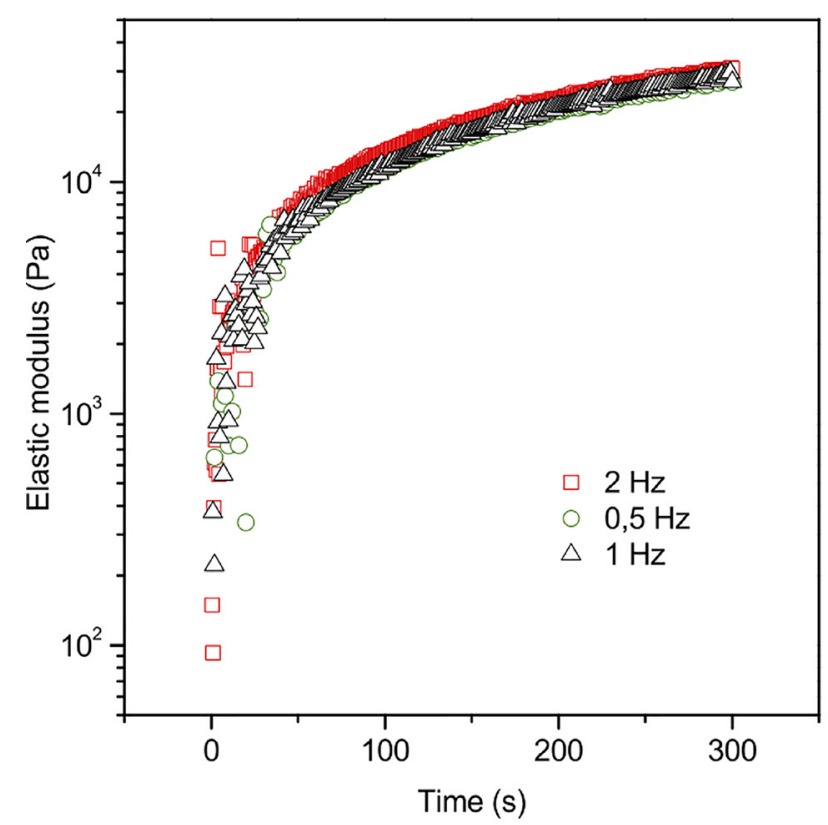

(a) Elastic modulus vs time as shown in Fig. 6(b). As we aim to measure the linear elastic modulus as a function of resting time in the range of [5-20] minutes, we chose $\gamma=10^{-4}\left(10^{-2} \%\right)$ which is in the linear domain for the three resting times. It can be seen in Fig. 6(b) that the data are scattered for small strain amplitude (typically $<0.01$ ) and for short resting time $(<20 \mathrm{~min})$. This is a classical problem encountered when a stress controlled rheometer is used to prescribe strain oscillations with small amplitude. This comes from the rheometer control loop which hardly manages to prescribe strain oscillations with small amplitude (less that $10^{-2} \%$ in our case) to materials with low stiffness $\left(<\right.$ a few $\left.10^{4} \mathrm{~Pa}\right)$. A strain amplitude equal to $10^{-2} \%$ appears to be a good compromise when measuring the elastic modulus of all of our suspensions.

As we worked with a stress controlled rheometer, an oscillatory shear stress is applied rather than an oscillatory shear strain, to avoid retro-action noise. To remain in the linear regime at a strain corresponding to $10^{-4}$, the stress applied on the sample must be updated to compensate for the evolution of the material properties over time. To do so, the measurement sequence is divided into periods of $10 \mathrm{~s}$; every $10 \mathrm{~s}$ the prescribed stress is adjusted based on the elastic modulus measured in the previous cycle so that the strain amplitude remains close to $10^{-4}$ (Fig. 7).

Typical results for the measured elastic modulus after various period of rest is depicted in Fig. 5. Measuring the evolution of elastic modulus as a function of time is a convenient way to characterize the evolution of the system microstructure during structuration of the suspension. Indeed oscillating strains do not induce microstructural rearrangement in the suspension as long as they remain in the linear domain. It has been checked that the strain oscillations we apply to the

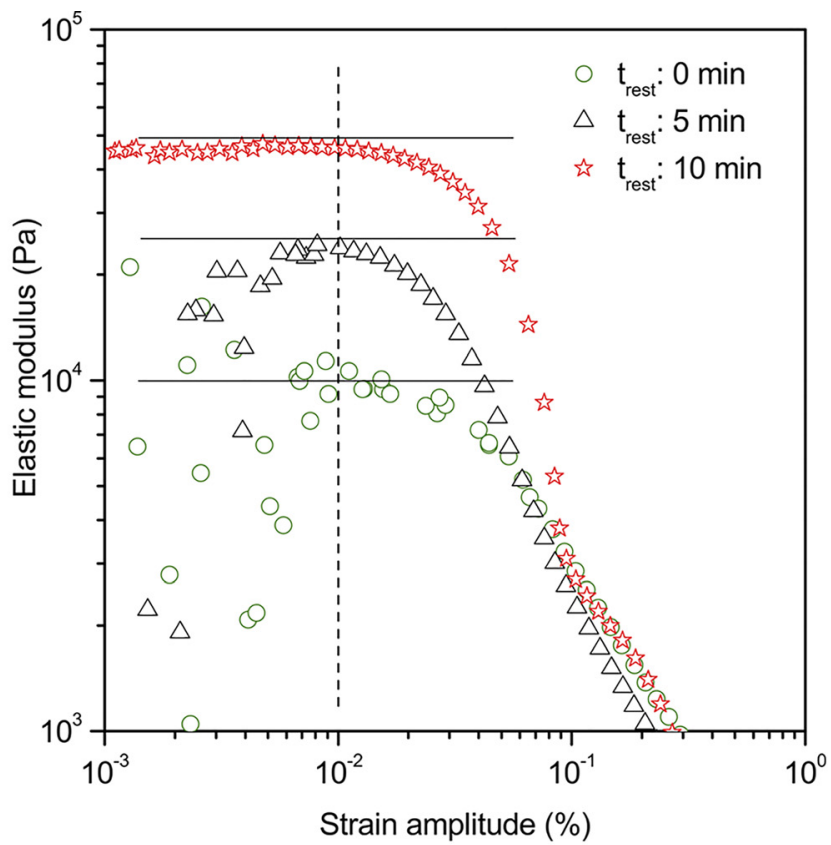

(b) Elastic modulus vs shear strain

FIG. 6. (a) Elastic modulus vs time for three different frequencies $(0.5,1$, and $2 \mathrm{~Hz})$ with $\gamma=10^{-4}$. (b) Elastic modulus vs shear strain for $2 \mu$ m crushed silica suspension with $\phi_{v}=0.37$ and $I=0.1$ for resting time $\mathrm{t}_{\text {rest }}=5,10$, and $20 \mathrm{~min}$. 
resting time $=\mathrm{n} \times 10 \mathrm{~s}$

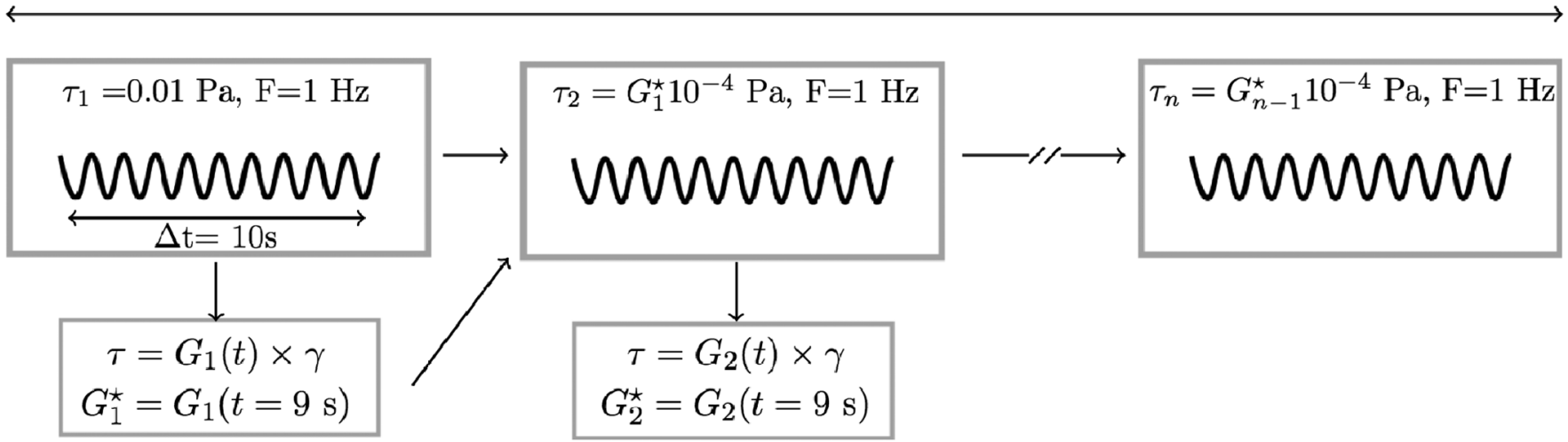

FIG. 7. The procedure we use to measure evolution of the elastic modulus as a function of time. F denotes the oscillatory shear stress frequency and $n$ is the number of 10 -s cycles in each sequence.

studied suspensions are nondestructive by comparing the yield stress measured after resting time for suspension with or without oscillations.

\section{Yield stress measurement method}

Yield stress is the minimum stress to initiate sample flow. As our suspensions are thixotropic, we observe reorganization of the microstructure at rest. To quantify the increase in yield stress during material aging, the yield stress is measured through a stress ramp. A linear stress ramp from 0 to $200 \mathrm{~Pa}$ in $2 \mathrm{~min}$ is applied on the suspension. We do not use the classical method, which consists in applying a small and constant shear rate to measure the yield stress because we observed that the control system of the rheometer is not accurate enough to impose a precise rotation of the inner tool. The ramp is automatically

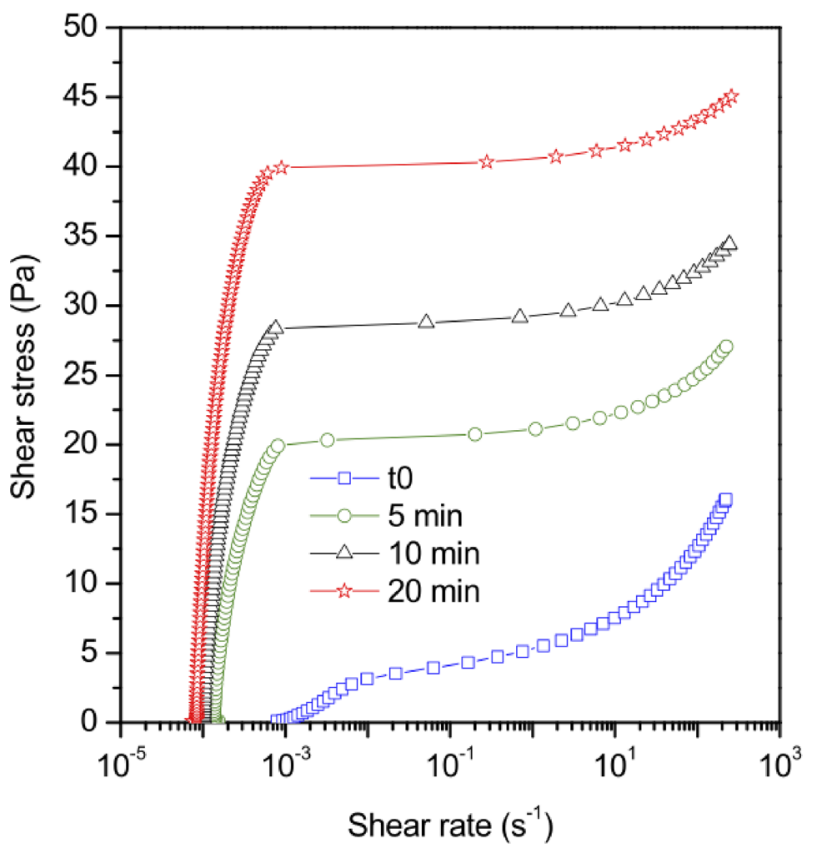

(a) Shear stress vs shear rate stopped once a shear rate of $200 \mathrm{~s}^{-1}$ is reached to avoid stronger destruction than during preshear. Figure 8 shows the yield stress measured on a suspension as a function of the shear rate (left picture) and as a function of the shear strain (right picture).

\section{Summary of the procedure}

First, quartz or silica bead suspensions are formulated with $\phi_{v}$ ranging from 0.3 to 0.4 and $I$ from 0.05 to 0.2 with the divalent salt $\mathrm{CaCl}_{2}$. The preparation procedure is carefully time controlled and the suspension is stirred with a vortex and sonicated in the Couette cup, before the geometry is put in the rheometer, so that the experiments start with a material as deflocculated as possible.

Once the material is in the rheometer, we apply the following loading history to the sample (Fig. 9 and Table III)

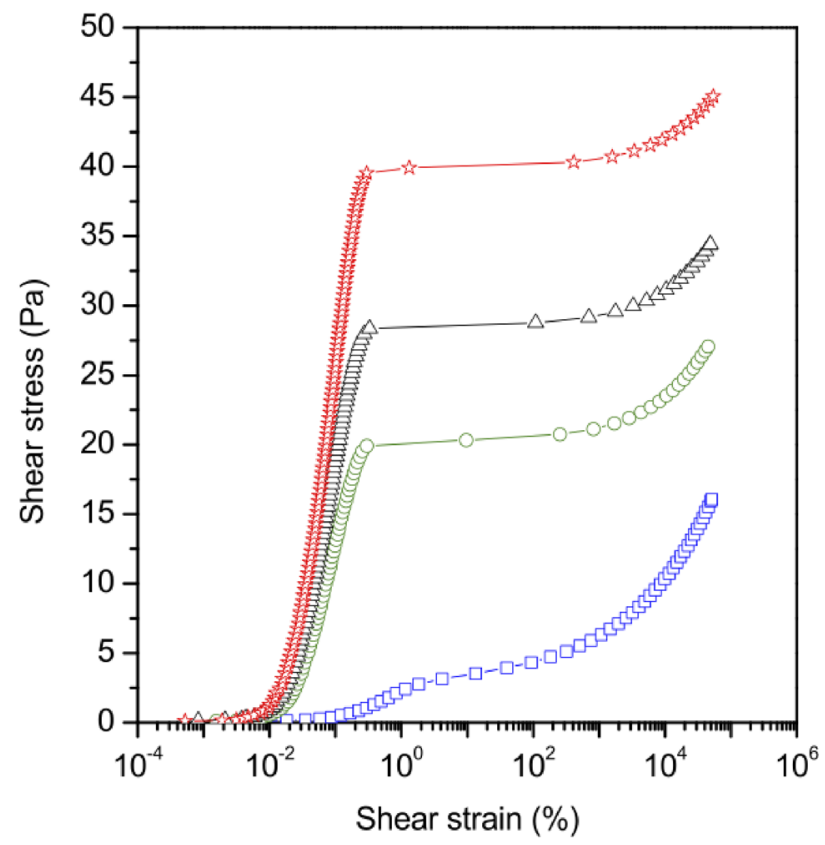

(b) Shear stress vs shear strain

FIG. 8. (a) Shear stress vs shear rate and (b) shear stress vs shear strain when a linear stress ramp is applied to suspension of $1.4 \mu$ m diameter silica beads at $\phi_{v}=0.38$ and $I=0.15$ for different resting times. 

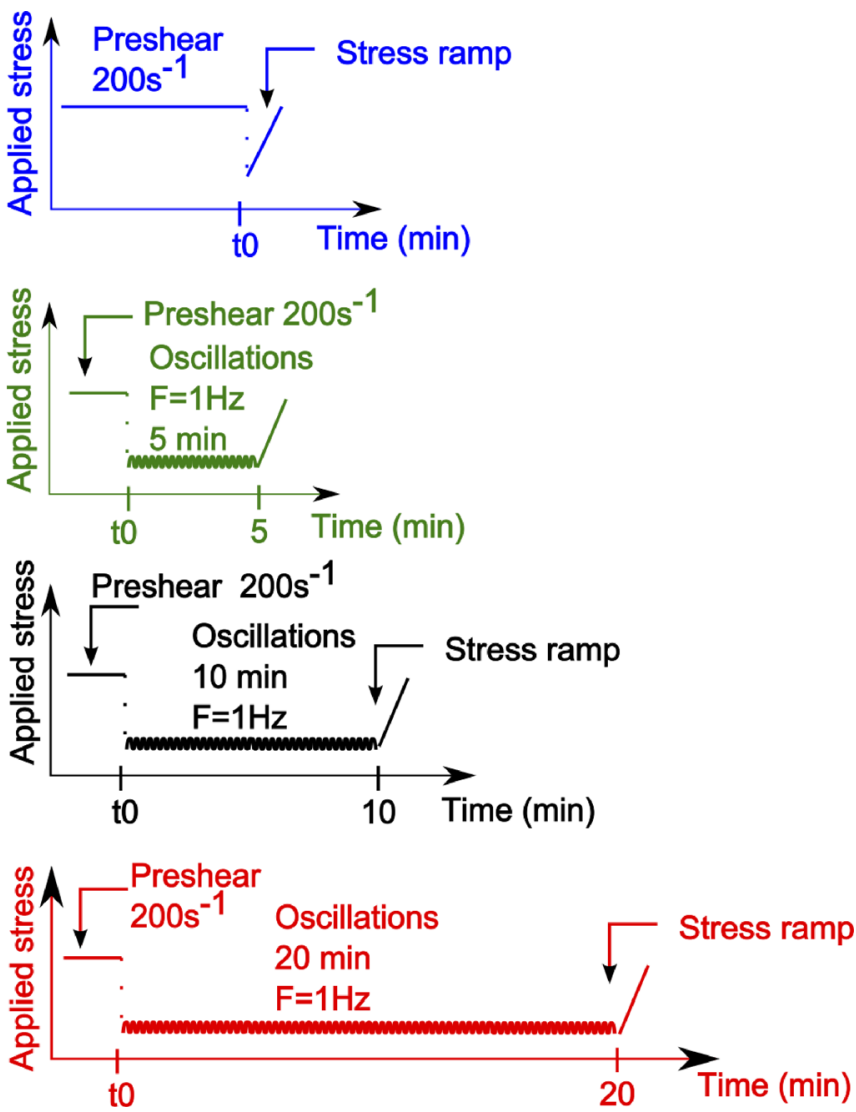

FIG. 9. Loading history applied to the sample during rheometric measurements.

where $t_{0}$ denotes the initial time of each loading sequence. It is reminded that the preshear durations (10 min after pouring the material into the rheometer and 3 min after measuring the suspension properties) have been chosen to ensure that the material is fully deflocculated at the beginning of each loading sequence.

TABLE III. Loading history applied to the sample during rheometric measurements.

1. The sample is strongly presheared at $200 \mathrm{~s}^{-1}$ for $10 \mathrm{~min}$ to get a reproducible initial state and eliminate the memory of pouring history.

2. Stress ramp from 0 to $200 \mathrm{~Pa}$ in 2 min allows to measure the yield stress at $\mathrm{t}_{0}$.

3. Preshear at $200 \mathrm{~s}^{-1}$ for $3 \mathrm{~min}$ to rejuvenate initial state.

4. Oscillatory shear stress during 5 min with $\gamma=10^{-4}$ and $\mathrm{F}=1 \mathrm{~Hz}$ to measure $G^{\prime}$ time-evolution during restructuration.

5. Stress ramp from 0 to $200 \mathrm{~Pa}$ in 2 min allows to measure the yield stress after $5 \mathrm{~min}$ at rest.

6. Preshear at $200 \mathrm{~s}^{-1}$ for $3 \mathrm{~min}$ to rejuvenate initial state.

7. Oscillatory shear stress during 10 min with $\gamma=10^{-4}$ and $\mathrm{F}=1 \mathrm{~Hz}$ to measure $G^{\prime}$ time-evolution during restructuration.

8. Stress ramp from 0 to $200 \mathrm{~Pa}$ in 2 min allows to measure the yield stress after $10 \mathrm{~min}$ at rest.

9. Preshear at $200 \mathrm{~s}^{-1}$ for $3 \mathrm{~min}$ to rejuvenate initial state.

10. Oscillatory shear stress during 20 min with $\gamma=10^{-4}$ and $\mathrm{F}=1 \mathrm{~Hz}$ to measure $G^{\prime}$ time-evolution during restructuration.

11. Stress ramp from 0 to $200 \mathrm{~Pa}$ in $2 \mathrm{~min}$ allows to measure the yield stress after $20 \mathrm{~min}$ at rest.

\section{RESULTS}

\section{A. Evolution of yield stress and elastic modulus}

\section{Silica beads}

In Fig. 10, we plot the evolution of the yield stress and the elastic modulus as a function of the particle volume fraction measured in suspensions of $1.4 \mu \mathrm{m}$ silica beads for different resting times and ionic strengths. Both the yield stress and elastic modulus of the suspensions increase with aging, ionic strength, and volume fraction. Increasing the ionic strength from $I=0.05$ to $I=0.1$ induces a large increase in both the yield stress and the elastic modulus (see experimental data for suspensions with volume fraction close to $39 \%$ ). A further increase in the ionic strength above $I=0.1$ seems to have less influence on the overall rheological properties of the suspension, especially for larger values of the solid volume fraction. This suggests that beyond a critical salt concentration adding salt has no more effect on flocculation of the suspension. The yield stress value increases strongly with the resting time while a slower increase is observed for elastic modulus (with the exception of suspensions with low ionic strength). This difference in behavior certainly comes from the difference in microstructural phenomena induced by the breaking (yield stress) and small reversible deformations (elastic modulus) of the particle network.

These experimental observations are consistent with microscopic theory. According to DLVO theory (see Sec. II), when salt is added to the suspending medium, the repulsive electrostatic potential is lowered and the amplitude of the repulsive barrier decreases. Then, the particles are more prone to van der Waals attraction. In the same way, the increase in volume fraction leads to a system more packed and more subject to aggregation. Both phenomena can explain the increase in the yield stress and the particle network stiffness. The increase in yield stress and elastic modulus with resting time is classically thought to be due to evolution of the particle network toward a more stable configuration induced by Brownian motion [5].

The increase in yield stress with ionic strength and volume fraction have already been reported by several authors $[8,18,20,21,24]$ while few studies deal with elastic modulus measurements. In mineral suspensions, many authors have shown that the addition of salt enhances flocculation, more often by measuring yield stress at different salt concentrations $[12,18,20,21,24,49]$. Franks [18] noticed the yield stress dependency with aging but without including details. Only a few studies have been devoted to the measurement of the evolution of the elastic modulus with time for thixotropic suspensions. Heiney et al. [17] have measured the evolution of the elastic modulus of silica suspensions with time and reported that the volume fraction greatly enhances the evolution rate.

Furthermore, simultaneous evolution of the yield stress and elastic modulus with resting time, which is of great interest for thixotropic materials as their structure evolves with time, has not been systematically studied either. As elastic modulus measurements are not destructive, it is easier to follow the time evolution of the sample by measuring $G^{\prime}$ rather 


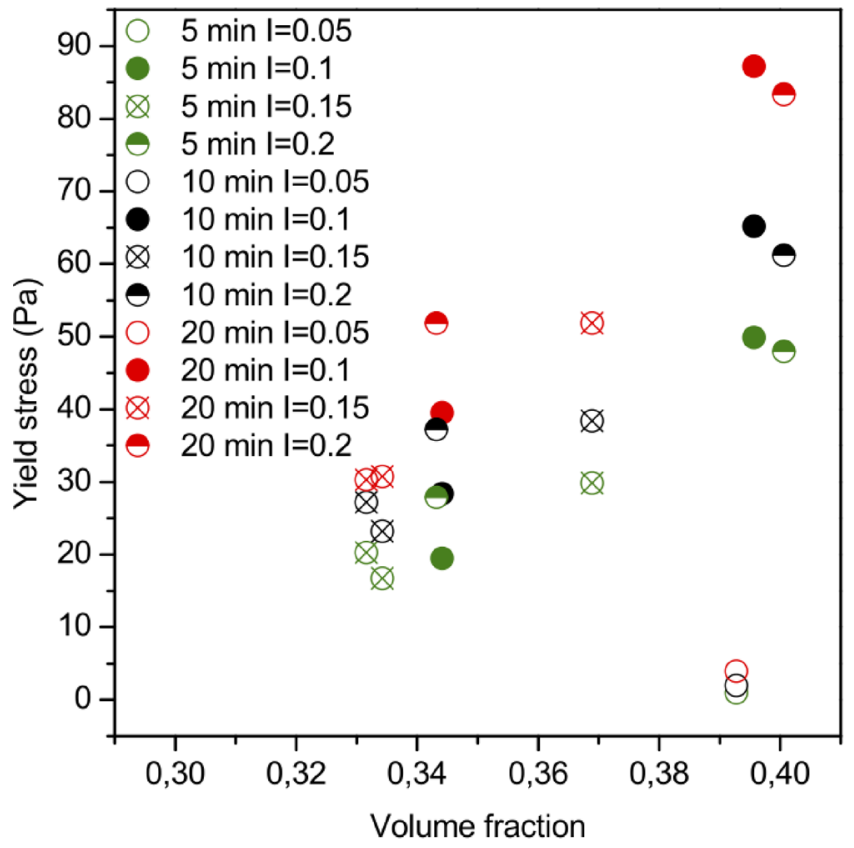

(a) Yield stress

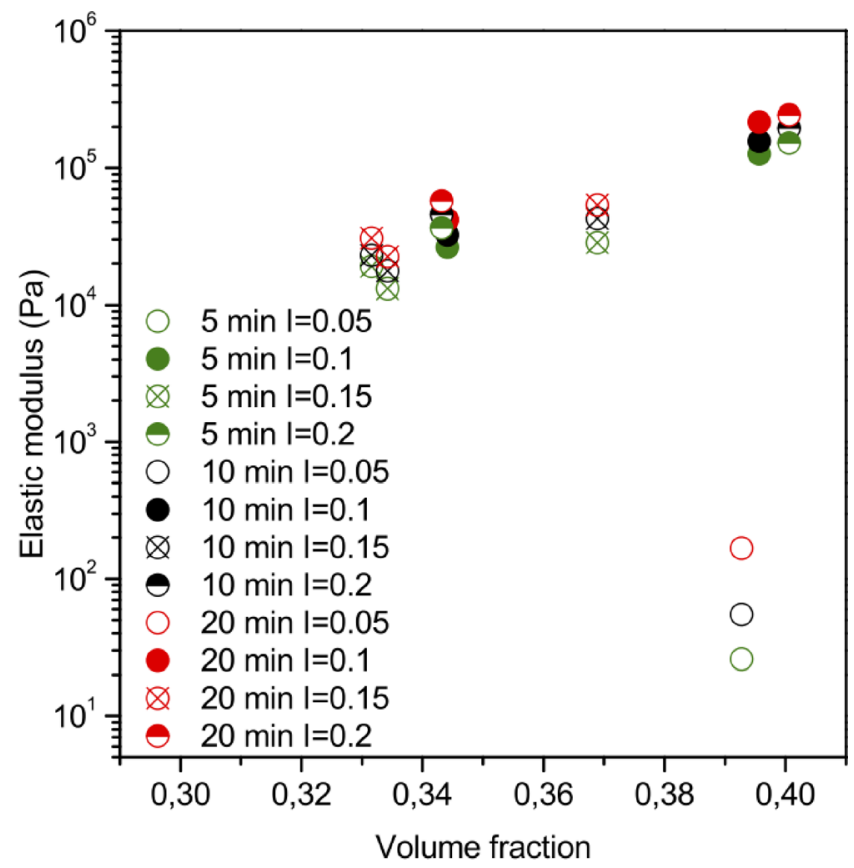

(b) Elastic modulus

FIG. 10. (a) Yield stress and (b) elastic modulus measured for $1.4 \mu \mathrm{m}$ silica bead suspensions as function of volume fraction for different times of rest and ionic strengths.

than by measuring $\tau_{c}$ at a given time. It is readily seen in Fig. 10 that the elastic modulus exhibits the same trend as the yield stress: $G^{\prime}$ is an increasing function of the ionic strength, the particle volume fraction, and the resting time. Then, it may be worthwhile investigating whether a relationship exists between the elastic modulus and the yield stress of colloidal suspensions. The important issue is addressed below.

As it can be seen in Fig. 11, the elastic modulus evolves over a very long time: We measure $G^{\prime}$ as a function of time for $10 \mathrm{~h}$ without reaching a plateau. It is likely that yield stress exhibits the same trend. Consequently, we decided not to study the long-term evolutions of the overall rheological properties of suspensions and to restrict our attention to the changes in elastic modulus and yield stress during the first 20 min after preshear has been stopped.

\section{Crushed silica}

The results obtained for crushed silica are presented in Fig. 12. As quartz particles are cheap and commercially available, reproducibility of the measurements can be tested easily. Thus, averaged results have been displayed in Fig. 12. One can notice that, except for few points, the reproducibility of the experiments is good.

As observed for the silica suspension, the elastic modulus and yield stress increase with resting time, particles volume fraction, and ionic strength up to a saturation point where an increase in concentration does not provide an additional effect on the system. The yield stress and elastic modulus seem to have a power law relationship with the volume fraction as already observed by several authors in literature $[9,15,50]$. It worth noticing that yield stress for the highest solid volume fraction is highly dispersed. This is a signature of the transition from a shear thinning behavior to a shear thickening behavior at low shear rate classically observed for colloidal suspension. This transition originates from the increase in frequency of contact between particles due to the increase in solid volume fraction. As we do not aim to study this transition, we restrict ourselves to solid volume fractions lower than $40 \%$ in this work. As regards the elastic modulus

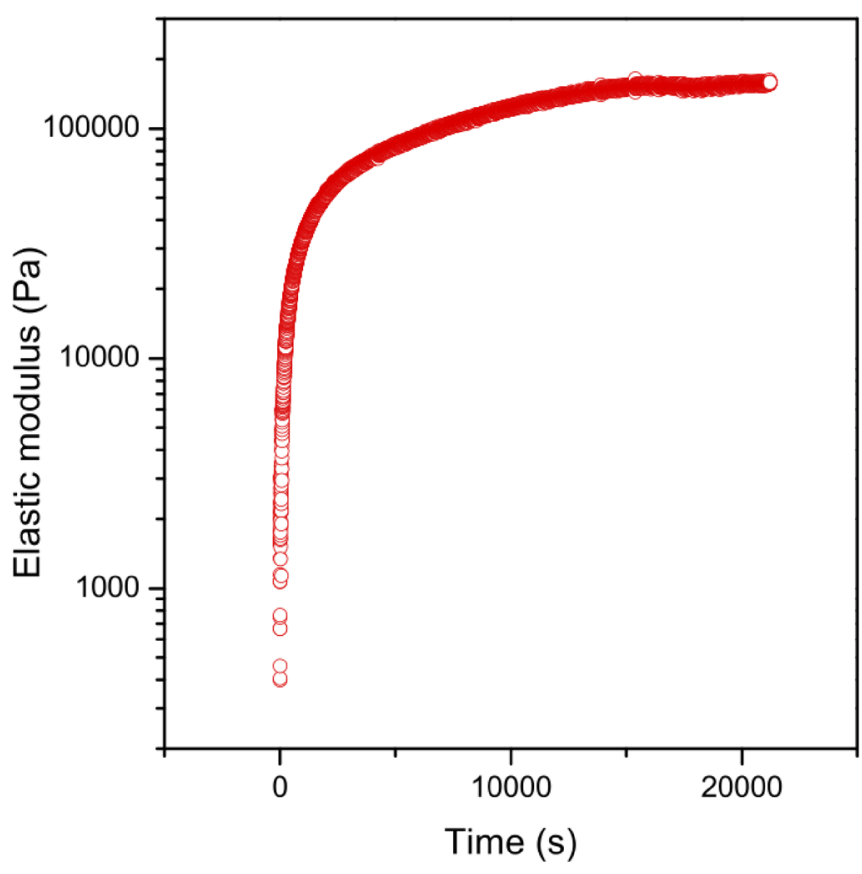

FIG. 11. Elastic modulus vs time for $2 \mu \mathrm{m}$ crushed silica with $\phi_{v}=0.36$ and $I=0.1$. 


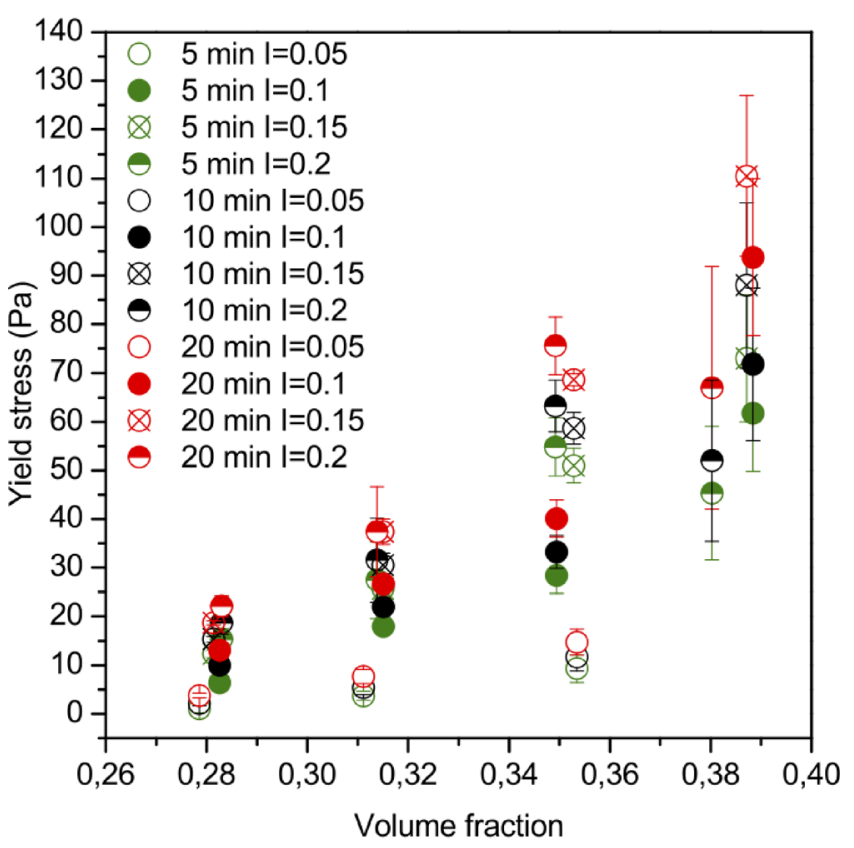

(a) Yield stress

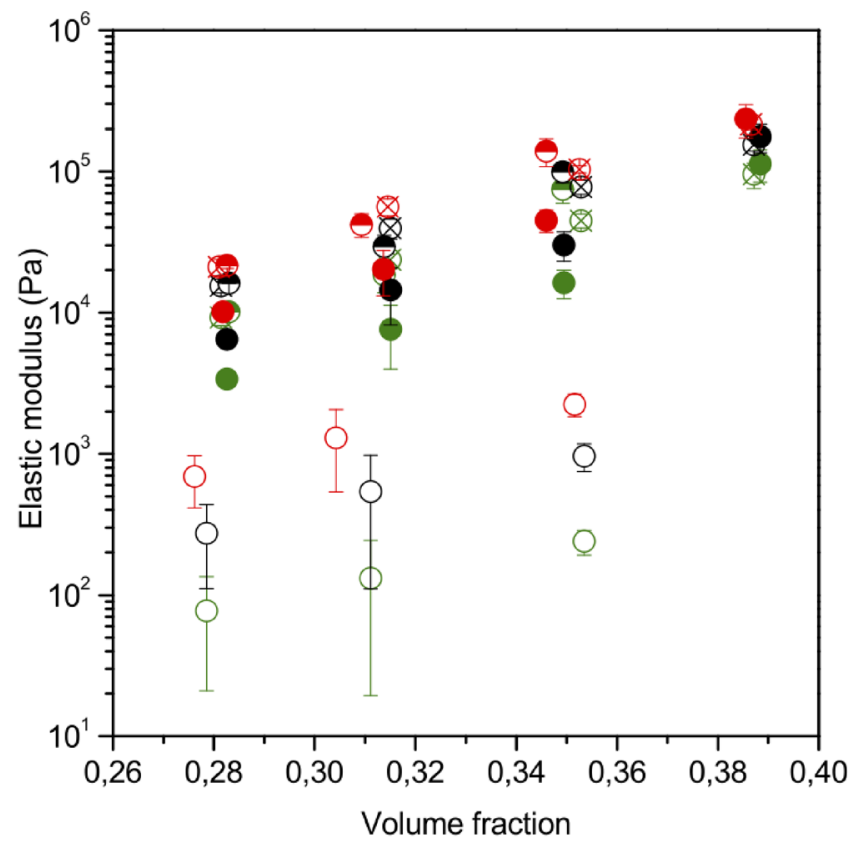

(b) Elastic modulus

FIG. 12. (a) Yield stress and (b) elastic modulus measured for $2 \mu \mathrm{m}$ crushed silica suspensions as function of volume fraction for different times of rest and ionic strengths.

measurements, experimental data are dispersed when the yield stress is low. In this situation, it is difficult to stay in the linear regime, even if the prescribed oscillatory strain amplitude is small. For example, the yield stress of the suspension with volume fraction approximately equal to $28 \%$ is close to a few Pascals whereas the elastic modulus is roughly equal to $10^{2} \mathrm{~Pa}$ to $10^{3} \mathrm{~Pa}$. In this situation, applying a shear strain amplitude of $10^{-4}$ can induce plastic deformation flawing the elastic modulus measurement. This problem goes away for larger values of the yield stress.

It is worth noting that Figs. 10 and 12 are very similar which suggests that angularity and polydispersity of the quartz particles do not give rise to different phenomena from that in play with silica beads. That might lead to the conclusion that trends and rules identified for well controlled silica bead suspensions remain true when dealing with less controlled systems such as crushed silica suspensions.

\section{B. Elastic modulus vs yield stress}

\section{Silica beads}

Both elastic modulus and yield stress depend on the suspension microstructure but provide different information. The elastic shear modulus describes the mechanical response of the system submitted to reversible small strain whereas yield stress is related to the breakage of the colloidal particle network. To check whether both quantities are linked and to demonstrate that $G^{\prime}$ can provide a reliable measurement of the reorganization and flocculation state of the suspension, we plot the elastic shear modulus as function of the yield stress in Fig. 13. As can be seen on these graphs, all the data collapse onto a single curve for a given particle size. One important point is that the same elastic modulus-yield stress couple can be measured for different formulations $\left(I, \phi_{v}\right)$, and loading history $\left(t_{\text {rest }}\right)$. For example, it can be seen in Fig. 13(b) that the red filled triangle corresponding to a suspension of $1.4 \mu \mathrm{m}$ particles with volume fraction $34.7 \%$, ionic strength 0.1 and resting time $20 \mathrm{~min}$ is very close to the black half filled triangle corresponding to a suspension of same particles with volume fraction $34.7 \%$, ionic strength 0.2 , and resting time $10 \mathrm{~min}$. It suggests that suspensions are in a "similar" state for the two different sets of data. It is worth noting that no scaling is used in Fig. 13. If a master curve exists on the elastic modulus-yield stress graph, it proves that both quantities are a macroscopic counterpart of the particle network state and that each of these quantities can equivalently characterize the suspension flocculation state. Then, they can be seen as structural parameters able to account for the flocculation state on the other macroscopic properties of the suspension.

If the elastic modulus can faithfully account for the evolution of the microstructure over time, then it may advantageously replace the dimensionless structural parameter $\lambda$ with no physical or experimental meaning usually used to build structural kinetics models [5].

Figure 13(b) depicts the results obtained for a suspension of $1.4 \mu \mathrm{m}$ particles with the same set of values for ionic strength, resting time, and solid volume fraction. The same trend is observed: couples of (elastic modulus-yield stress) values for different systems collapse onto a single curve. It is worth noting that for the same formulation (volume fraction, ionic strength, and resting time), the smallest particles give a larger yield stress and to a lesser extent a larger elastic modulus. On the contrary, Fagan and Zukoski [8] found that the 


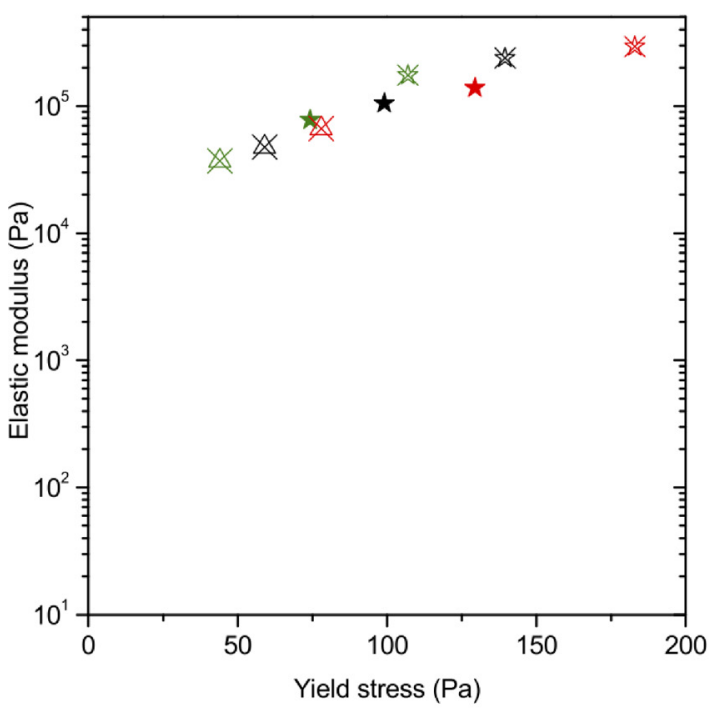

(a) $1.0 \mu \mathrm{m}$

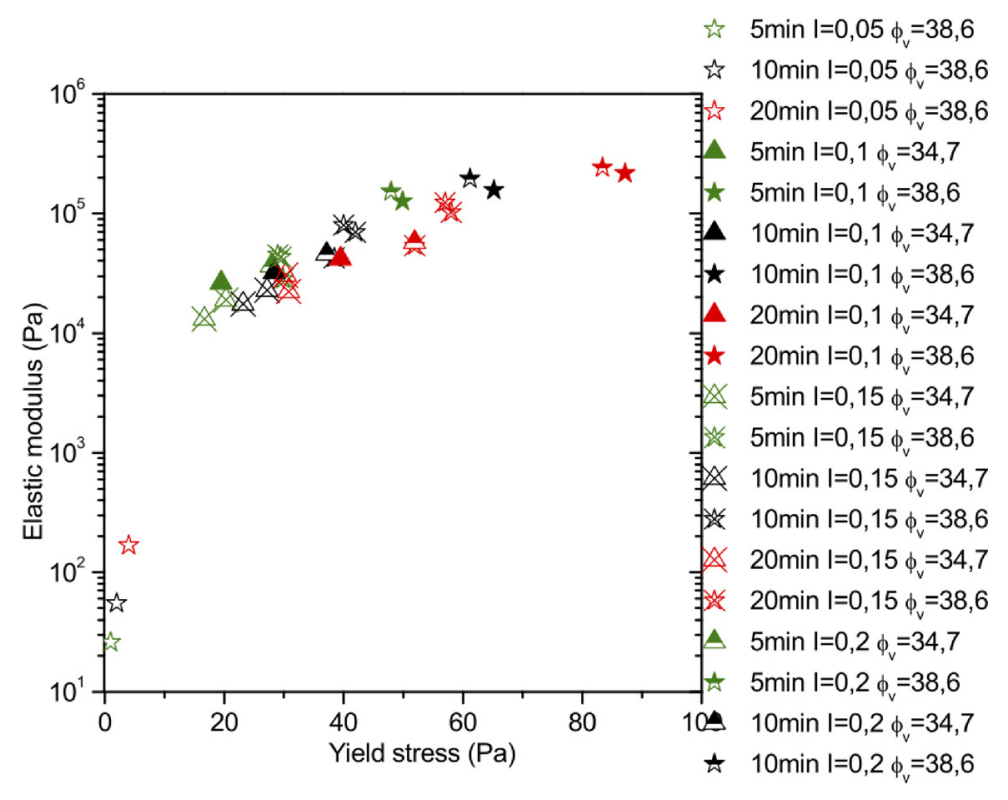

(b) $1.4 \mu \mathrm{m}$

FIG. 13. Elastic modulus as function of yield stress for all formulation prepared with (a) $1.0 \mu \mathrm{m}$ and (b) $1.4 \mu \mathrm{m}$ silica beads.

elastic modulus of suspensions made of silica beads synthesized via a Stöber process increases with particle size. However, other researchers have also measured an increase in elastic modulus when reducing the size of the silica particles [20]. This issue is discussed further in Sec. IV.

\section{Crushed silica}

Similar data measured for crushed silica suspensions are plotted in Fig. 14. For the sake of readability, the legend is

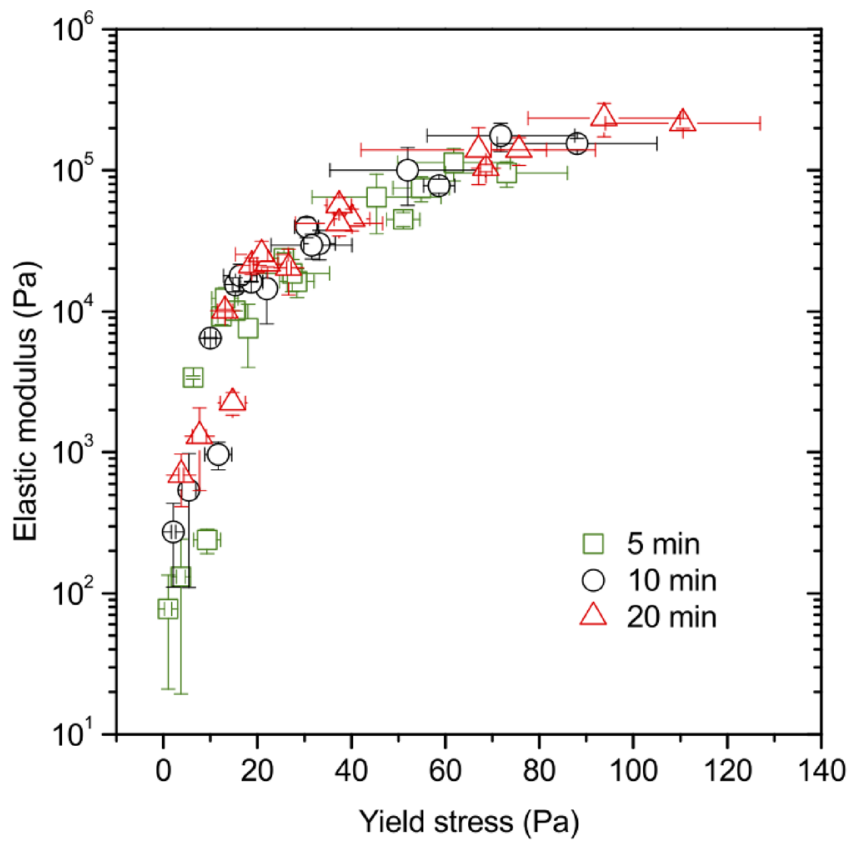

(a) $2 \mu \mathrm{m}$ not fully detailed because of the huge number of experiments done on this system. But as for the silica beads the data in Fig. 14 has been measured for suspensions with a solid volume fraction ranging from $30 \%$ to $40 \%$, an ionic strength from 0.05 to 0.2 and a resting time equal to 5,10 , or $20 \mathrm{~min}$. For each mean size of quartz particles, the elastic modulus versus yield stress data collapse onto a single curve. We also observe that yield stress and elastic modulus of crushed silica suspensions are decreasing function of the median size of the particle. Despite the material is much less controlled, i.e., the

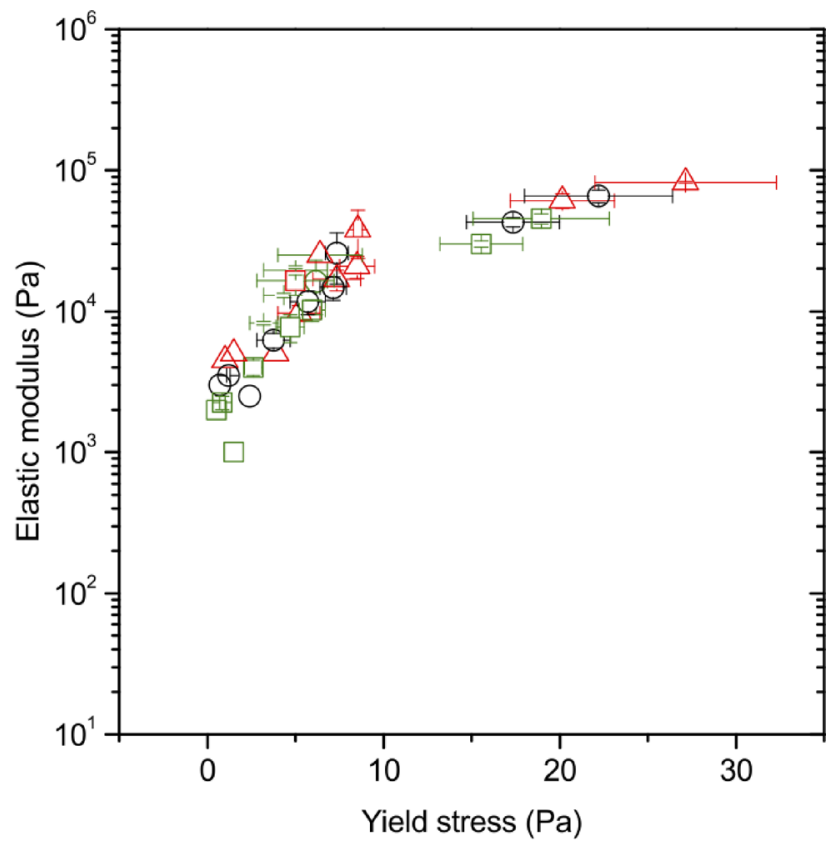

(b) $4 \mu \mathrm{m}$

FIG. 14. Elastic modulus vs yield stress for the crushed silica suspensions we studied for the range of parameters $\left(I \in[0.05,0.2]\right.$ and $\left.\phi_{v} \in[0.28,0.40]\right)$ with (a) $2 \mu \mathrm{m}$ and (b) $4 \mu \mathrm{m}$ particles. 
particles are polydisperse and have sharp-edges, the same trends as for silica monodisperse beads are observed. This confirms that the interplay of the colloidal interactions, solid volume fraction, and resting time control the overall properties of crushed silica suspensions in the same way as for monodisperse silica bead suspensions.

\section{Size effect}

We plot in Fig. 15 the elastic modulus as a function of the yield stress for all the silica bead suspensions [Fig. 15(a)] and all the crushed silica particle suspensions [Fig. 15(b)] we studied. We obtain one curve for each particle size: Both the elastic modulus and the yield stress of the suspension depend on the particle size. Owing to the narrow size distribution of silica beads (see Fig. 1), the offset between the curves $G^{\prime}=\mathrm{f}\left(\tau_{c}\right)$ we obtain for the silica bead suspensions can only be due to a difference in particle size. For crushed silica suspensions, polydispersity of the particles could have masked the mean size effect on the elastic modulus-yield stress relationship. The fact that we obtain two different curves when plotting the suspensions elastic modulus as a function of the yield stress in Fig. 15(b) proves that the mean size of the particles is still a key parameter in this case. Of course, this result should be valid only for suspensions with low polydispersity.

\section{Resting time}

Rheometry measurements clearly show that the yield stress and elastic modulus depend on the resting time. To check if these time evolutions are due to some change of the suspension microstructure, we observed the silica bead suspensions with a confocal microscope LSM 700 Zeiss with an immersive objective $\times 100$ Plan-APOCHROMAT Zeiss. To

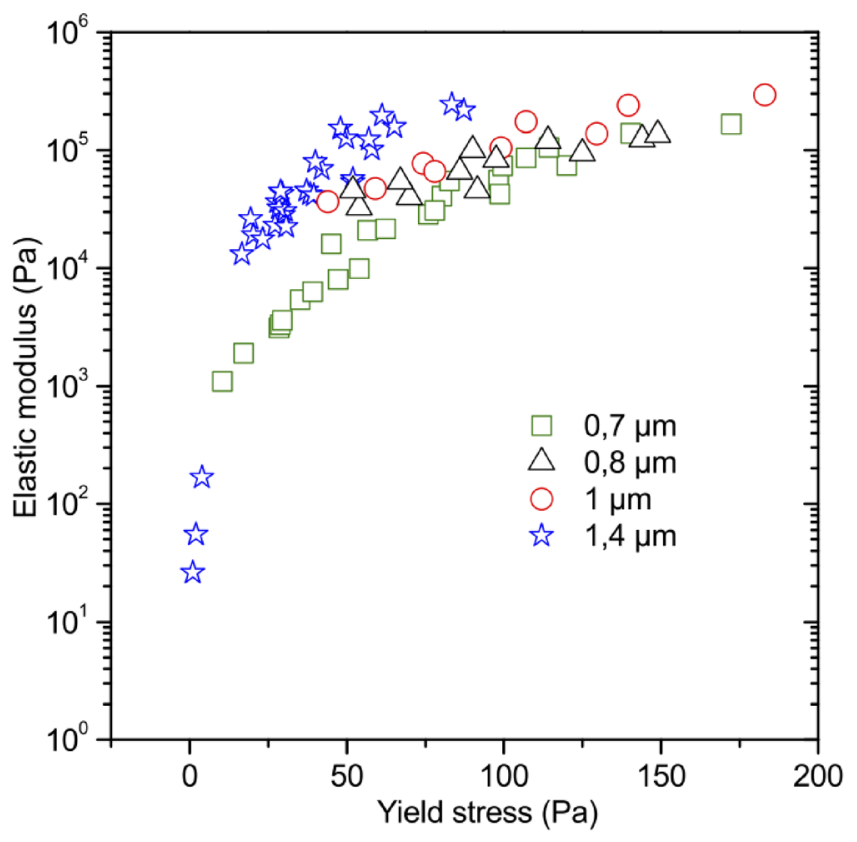

(a) Silica beads allow deeper penetration of the laser into the samples, and to avoid end effects which can be observed near the bottom wall, the optical index of the suspending medium is partially matched to that of the silica particles with the addition of 40 wt. \% of glycerol. Even if the addition of glycerol slightly modifies the rheological properties of the suspending fluid, we checked that suspensions with the matched optic index behave in the same way as the original suspensions: Yield stress and elastic modulus increase with ionic strength, resting time, and solid volume fraction.

The suspension is deposited in a rectangular tank, the bottom of which is a glass lamella and covered to avoid evaporation during experiments (Fig. 16). It was not possible to control and apply exactly the same preshear as in the rheometer to the sample. Nevertheless the sample is strongly presheared and destructured manually with a spatula before observations.

The rheological properties of a microstructured suspension of $1.4 \mu \mathrm{m}$ silica beads with $\phi_{v}=0.39, I=0.15$, and $40 \mathrm{wt}$. \% glycerol have been measured. Its microstructure has been observed using confocal microscopy. It appears clearly in Fig. 17 that even if the elastic modulus increases with resting time over 2 orders of magnitude, no structural changes are seen in the series of pictures taken over a period of $10 \mathrm{~min}$. A careful examination of the four pictures of the suspension reveals that particles do not move. Differences between the images come only from the contrast level of exposure time variations.

At this stage, flocculation and thixotropy may be summarized as follows:

- First, a primary structure is almost instantaneously formed when an external load is released. Particles' aggregation should be very fast assuming that each binary collision

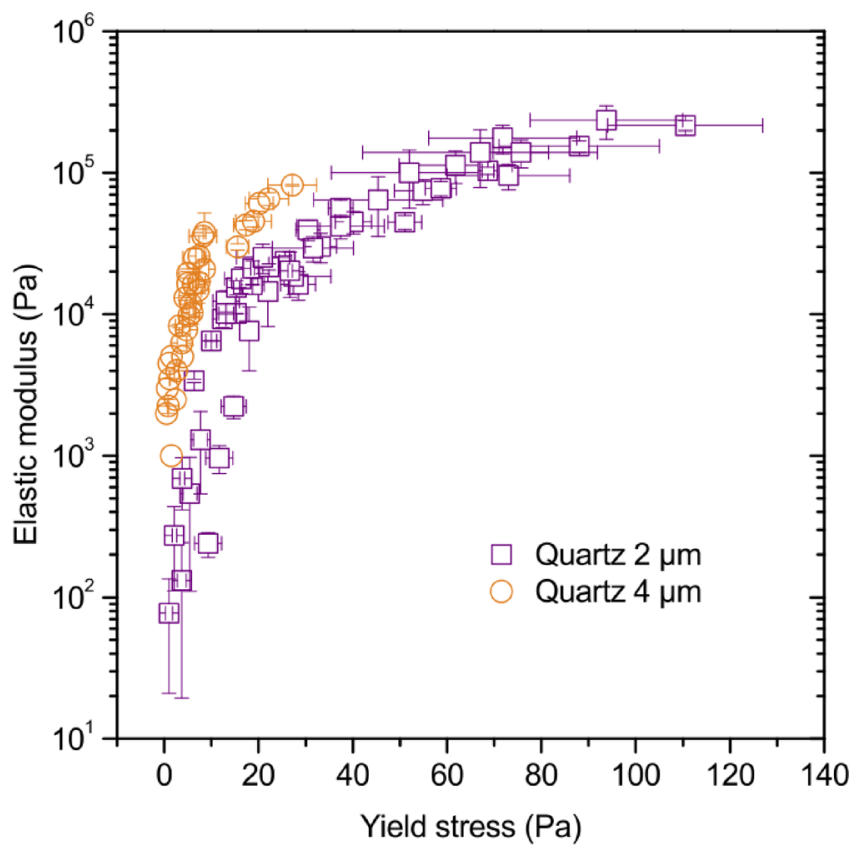

(b) Crushed silica (Quartz)

FIG. 15. (a) Elastic modulus vs yield stress for all different sizes silica beads and (b) for crushed silica with two mean diameters. 
glass cover to avoid evaporation

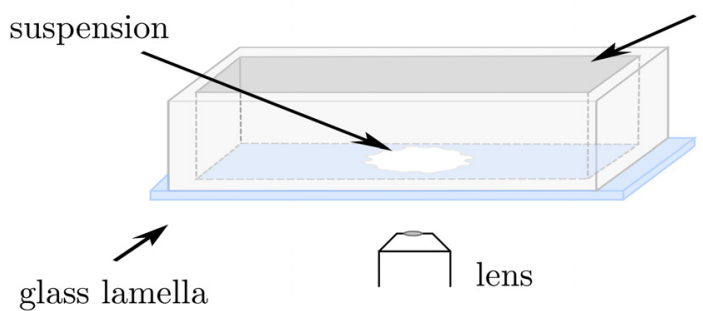

$\mathrm{e}=160 \mu \mathrm{m}$

FIG. 16. Confocal microscopy tank.

causes the two particles to stick together. The rate of doublet formation was calculated by Von Smoluchowski [51]

$$
J_{0}=\frac{8 k_{B} T n^{2}}{3 \mu}
$$

with $n$ as the particle density

$$
n=\frac{3 \phi_{v}}{2 \pi d_{p}^{3}} .
$$

Putting $\phi_{v}=0.3$ and $d_{p}=1.4 \mu \mathrm{m}$ into Eqs. (5) and (6) yields $n \approx 10^{17}$ particles $/ \mathrm{m}^{3}$ and $J_{0} \approx 10^{17}$ doublets formed $/ \mathrm{m}^{3}$ per second, which means that particles agglomerate in few seconds. The results of this rough calculation are consistent with experimental observations.

Even if the evolution of the suspension microstructure cannot be detected, very different microstructures are initially formed and observed when the suspension formulation is varied as can be seen in Fig. 18 for two suspensions of silica beads with the diameter $1.4 \mu \mathrm{m}$, solid volume $35 \%$, and two different ionic strength $I=0.05$ and $I=0.1$. Then, this primary structure depends on the formulation of the suspension and, in particular, on the interparticle force intensity depending on the ionic strength.

- Once the primary structure has been formed, thixotropy arises without detectable or noticeable microstructural evolution as evidenced in Fig. 17. Therefore, the evolution of the overall rheological properties can be explained in two ways. On the one hand, Brownian agitation produces particle displacements of a few nanometers that cannot be detected with a conventional microscope but which induces changes in the interparticle forces. On the other hand, changes in interparticle forces (tangential or normal) arising from chemical bonding or other physico-chemical phenomena occurring close to the contact between particles that modify the overall rheological properties without any evolution of the microstructure.

This question is further discussed in Sec. IV.

\section{DISCUSSION ABOUT MECHANISMS INVOLVED IN THIXOTROPY}

To our best knowledge, no experimental technique (or hardly accessible) allows one to probe such small particle displacement or to measure forces between particles (less than $5 \mu \mathrm{m}$ ) suspended in a fluid. Therefore, it is impossible to directly assess the relevance of the explanations proposed

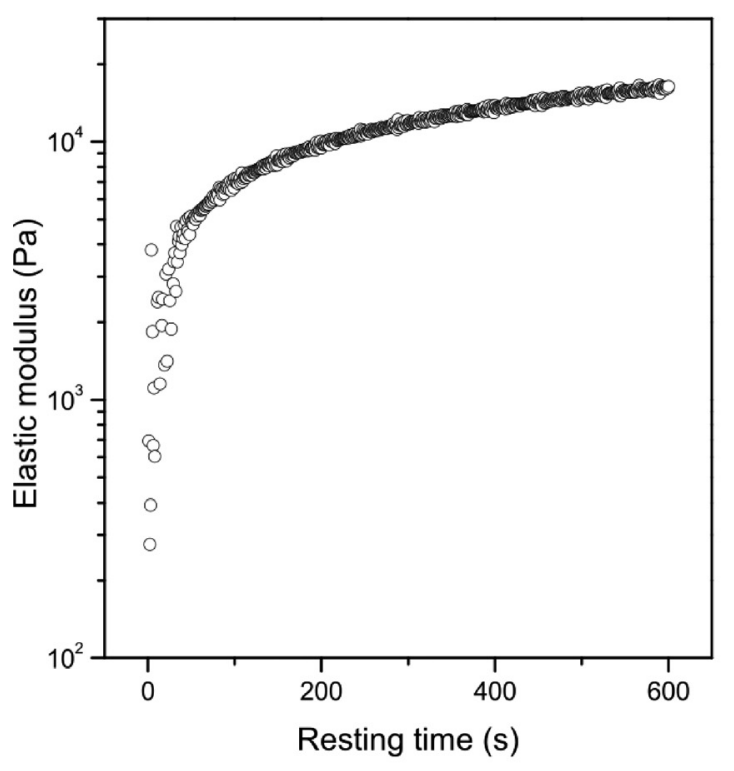

(a) Elastic modulus

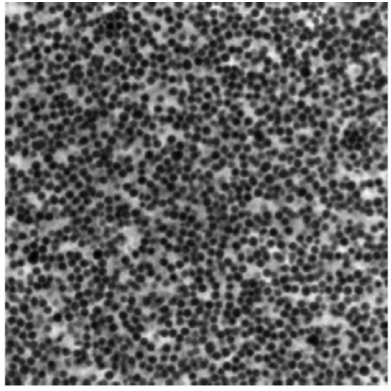

(b) $t_{0}$

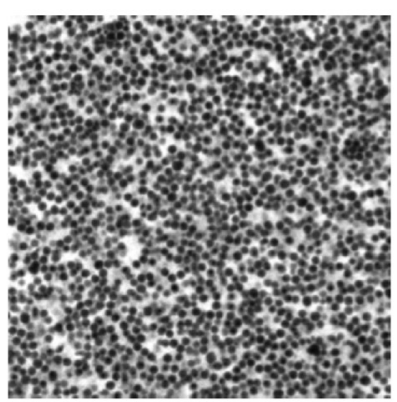

(d) $5 \mathrm{~min}$.

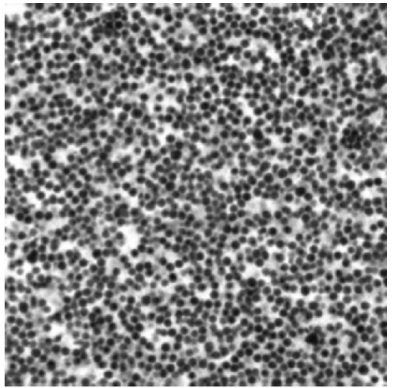

(c) 2 min.

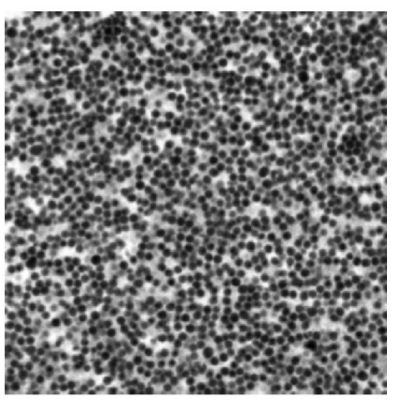

(e) $10 \mathrm{~min}$.

FIG. 17. Comparison of the elastic modulus (a) and the microstructure (b)-(e) evolutions during aging of $1.4 \mu \mathrm{m}$ silica bead suspension at $39 \%$ volume fraction, $I=0.15$, and $40 \mathrm{wt}$. $\%$ glycerol in suspending medium. 


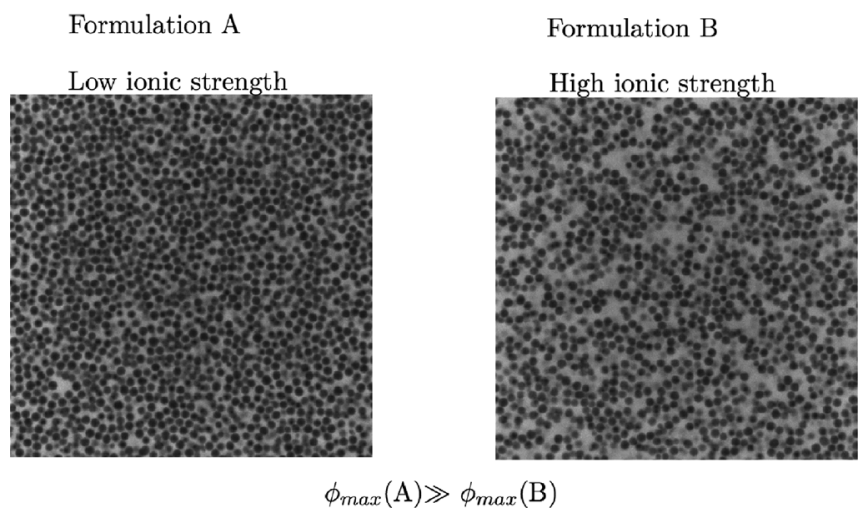

FIG. 18. Microstructure of two $1.4 \mu \mathrm{m}$ silica bead suspensions with $\phi_{v}=0.35$ low $(I=0.05$, left $)$ and high $(I=0.2$, right $)$ ionic strength observed with a confocal microscope. Both suspensions have exactly the same solid volume fraction, whereas microstructures are rather different.

below. However, theoretical models which predict the overall rheological properties of a colloidal suspension, from the description of its microstructure and the interparticle forces, have been proposed in the literature. We propose to compare the predictions of these models to our experimental data in order to verify which effect can explain our experimental findings. In this framework, studying how the rheological properties of the suspension depend on the particle size should make it possible to assess which interpretation is realistic.

\section{A. Brownian motion}

Thixotropy encountered in colloidal suspensions can be explained through Brownian motion. Indeed particles submitted to Brownian agitation continually explore different configurations which allow them to reorganize in more energetically favorable configurations [4,5]. These movements might not be detected experimentally. Indeed regarding DLVO potentials depicted in Fig. 19 a few nanometers displacement is enough to considerably change the interactions between particles. As can be seen in Fig. 19, the distance separating the maximum and the secondary minimum of the DLVO potential is $3 \mathrm{~nm}$ for $0.7 \mu \mathrm{m}$ silica beads suspended in a solution with an ionic strength $I=0.05$, which means that the particles displacement of $1 \mathrm{~nm}$ may induce a huge change in the interparticle force.

\section{Models}

Most models we found in the literature concerning the behavior of colloidal suspensions are based on the assumption that interparticle forces are central and can be described by DLVO theory $[34,35]$. It is out of the scope of this paper to review in detail all these models; it is enough to say that a model does not exist where there is a broad agreement. Most of the existing models contain adjustable parameters or functions such as the interparticle distance $h[10,15,27]$, the maximum packing fraction $\phi_{m}$ [10], the percolation threshold $\phi_{0}$ [10], the coordination functions $K\left(\phi_{v}\right)$ [27], chosen so that the model accurately fits the experimental data.

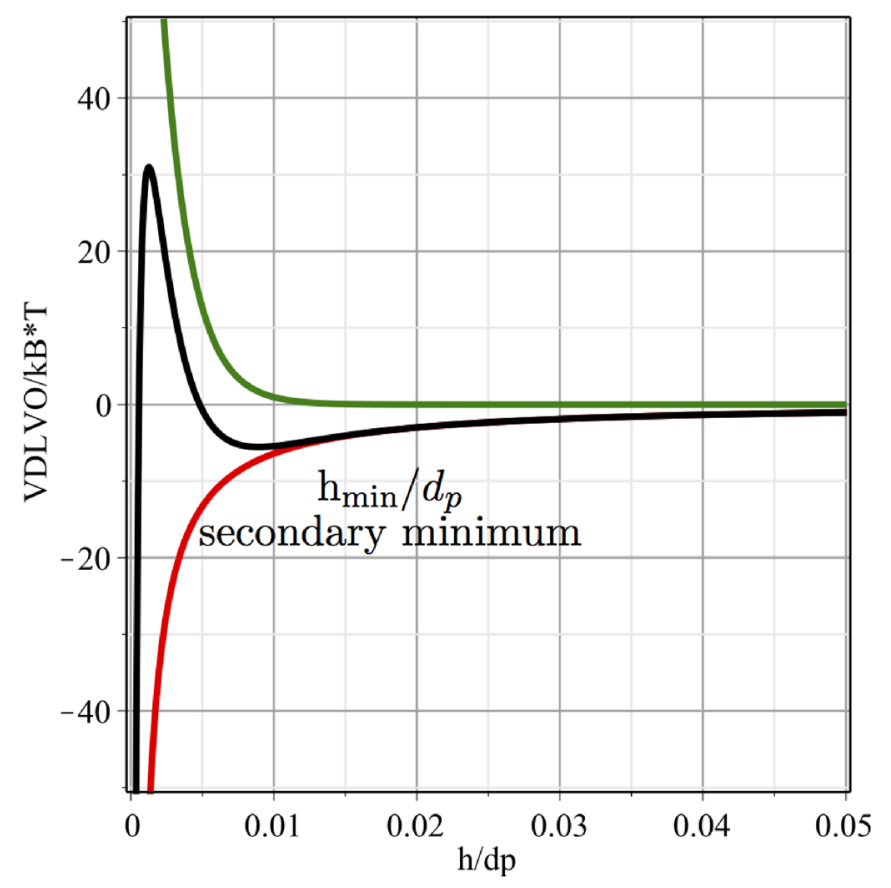

FIG. 19. Dimensionless DLVO potentials for $0.7 \mu \mathrm{m}$ silica beads interacting in a solution at $I=0.05$ with $A_{H}=3.4 \times 10^{-21} \mathrm{~J}$, and $\Psi_{0}=-15 \mathrm{mV}$.

We have gathered the estimates for the yield stress of three models in Table IV and the estimates for the elastic modulus of two models in Table V.

The two estimates for the elastic modulus and the Russel et al. [2] estimate for the yield stress are valid no matter the interaction potential $V_{\text {int }}$. While there is no adjustable parameter in the Russel et al. [2] model, the Buscall estimate for the elastic modulus depends linearly on a coordination number $N$ that cannot be directly evaluated. In the framework of this work, it makes sense to use the simplified expression of the DLVO potential [Eq. (2)] for two spheres immersed in an asymmetrical electrolyte as an interaction potential. The Flatt and Bowen model takes into account van der Waals, electrostatic, and steric forces. Three parameters are used as adjustable variables: The maximum packing fraction $\phi_{m}$, the percolation threshold $\phi_{0}$, and the minimum interparticle separation distance $h_{\mathrm{FB}}$. Finally, the Scales et al. model for the yield stress relies on the simplified Eq. (2) for the interparticle interaction and the mean interparticle separation distance $h$ depending on a particle crowding factor $X_{0}$ and a characteristic separation distance $h_{0}$ which are fitting parameters.

TABLE IV. Models from literature expressing yield stress as a function of potential of interaction in colloidal systems.

\begin{tabular}{|c|c|c|}
\hline References & Models & Fitting parameters \\
\hline Scales et al. [27] & $\tau_{c}=\frac{\phi_{v}}{24 \pi} \frac{K\left(\phi_{v}\right)}{d_{p}}\left(\frac{A_{H}}{h^{2}}-\frac{24 \pi \varepsilon \varepsilon_{0} \kappa \Psi_{0}^{2}}{(1+\exp (\kappa h)}\right)$ & $\begin{array}{c}h=h_{0} X_{0} \\
\exp \left(-4.5 \phi_{v}\right) \\
h_{0}, X_{0}\end{array}$ \\
\hline $\begin{array}{l}\text { Flatt and } \\
\text { Bowen [10] }\end{array}$ & $\tau_{c}=\frac{1.8}{\sqrt{3} \pi^{3}}\left(\frac{A_{H}}{12 h_{\mathrm{FB}}^{2} d_{p}}\right) \phi_{v} \frac{\phi_{v}^{2}\left(\phi_{v}-\phi_{0}\right)}{\phi_{m}\left(\phi_{m}-\phi_{v}\right)}$ & $\phi_{m}, \phi_{0}, h_{\mathrm{FB}}$ \\
\hline Russel et al. [2] & $\tau_{c} \approx \frac{\phi_{v}^{2}}{d_{p}^{2}}\left(\frac{\partial V_{\mathrm{int}}}{\partial h}\right)_{\max }$ & \\
\hline
\end{tabular}


TABLE V. Models from literature expressing elastic modulus as a function of potential of interaction in colloidal systems.

\begin{tabular}{lcc}
\hline \hline References & Models & Fitting parameters \\
\hline Buscall [15] & $G=\frac{\alpha}{h} \frac{\partial^{2} V_{\text {int }}}{\partial h^{2}}$ & $\alpha=\frac{3}{32} \phi_{m} N$ \\
Russel et al. [2] & $G \approx \phi_{v}^{2} \frac{k_{B} T}{d_{p}^{2}}\left[-\frac{\left(V_{\text {int }}\right)_{\min }}{k_{B} T}\right]^{\frac{3}{2}}$ & \\
{$\left[\frac{1}{k_{B} T}\left(\frac{\partial^{2} V_{\text {int }}}{\partial h^{2}}\right)_{\text {min }}\right]^{\frac{1}{2}}$} & \\
\hline \hline
\end{tabular}

It is worth noting that both Scales et al. and Flatt and Bowen estimates for the yield stress scale inversely with the size of the particle. The same scaling is obtained when the simplified Eq. (2) is put into the Russel et al. [2] model. With regard to the elastic modulus, putting the simplified DLVO potential [Eq. (2)] into the estimates of Table V yields expressions that do not depend on the particle size.

Before paying attention to alternative models for the overall properties of colloidal suspensions, it is advisable to recall that several authors have reported experimental data or arguments that contradict the predictions of these models.

\section{Limits}

Some authors [6,52,53] have measured forces curves between smooth mica surfaces with different ionic aqueous solution, and they have shown that the experimental data do not agree with the DLVO theory, especially for short interparticle distance. Solvation forces [12,49], ion-correlation forces [54-56], or steric forces are commonly discussed, but no general expression for these forces has been proposed. If we look into the details of our experimental results (see Table VI), it is obvious that the elastic modulus depends on the particle size, as already observed by Chen et al. [20]. On the contrary, models relying on the DLVO description of the interparticle force predict that the elastic modulus does not depend on the particle size. More precisely, models based on DLVO theory and Brownian motion predict that the elastic modulus does not depend on the particle size and that overall rheological properties stop evolving after a few seconds of resting time, which disagrees strongly with our experimental results. Such models do not apply to the silica suspensions we study.

\section{B. Interparticle bonds}

Other models based on contact theory have been investigated. Pantina and Furst [57] have seen the presence of tangential forces between colloidal particles in suspension through bending experiments. This contradicts previous models based on DLVO interactions which are centrosymmetric and consequently assume free rotation of the particles.

\section{Models}

Based on the Johnson, Kendall, and Roberts (JKR) theory of adhesion [58] and assuming that the suspensions elastic modulus depends on the bending rigidity of the largest cluster, a scaling relation for the elastic modulus of a fractal network can be derived [57]

$$
G=\frac{2 k_{0}}{d_{p}} \phi_{v}^{\left(3+d_{b}\right) /\left(3-d_{f}\right)}
$$

with $k_{0}$ denoting the bending rigidity of a single colloidal bond, $d_{b}$ as the bond dimension and $d_{f}$ representing the fractal dimension of the gel. It leads to $G \approx K d_{p}^{-4 / 3}$ with $K$ accounting for solid liquid surface energy and the elastic property of the particle constitutive material.

During bending experiments on particles, Pantina and Furst [57] observed local rearrangements of the particles occurring when the bending moment is larger than a critical value $M_{c}$ which characterizes the onset of microstructural yielding. By assuming that the critical stress for the onset of plastic deformation (yield) of the suspension is the stress required to induce local rearrangements at the particle scale, it can be shown that

$$
\tau_{c} \approx M_{c} d_{p}^{3}{\frac{\phi_{v}}{\phi_{e}}}^{3 /\left(3-d_{f}\right)}
$$

where $\phi_{e}$ denotes an effective cluster volume fraction. Pantina and Furst found $M_{c} \propto d_{p}^{2}$ which leads to $\tau_{c} \propto d_{p}^{-5 / 3}$ [57].

\section{Limits}

Even if this model predicts that both the elastic modulus and the yield stress depend on the particle size, it is based on assumptions that deserve to be discussed. First, the relationship between the microscopic properties and rheological behavior is developed based on contact theory. Although experimental force measurements point out a very strong repulsion at a short distance [6] that may prevent direct contact between the particles. Accounting only for contact between solid surfaces in colloidal silica suspensions may also be debated, particularly regarding that chemical bonds

\begin{tabular}{|c|c|c|c|c|c|c|c|c|}
\hline \multirow[b]{3}{*}{ Diameter $(\mu \mathrm{m})$} & \multirow[b]{3}{*}{ Volume fraction } & \multirow[b]{3}{*}{ Ionic strength } & \multicolumn{3}{|c|}{ Yield stress $(\mathrm{Pa})$} & \multicolumn{3}{|c|}{ Elastic modulus $(\mathrm{Pa})$} \\
\hline & & & \multicolumn{3}{|c|}{ Resting time (min) } & \multicolumn{3}{|c|}{ Resting time (min) } \\
\hline & & & 5 & 10 & 20 & 5 & 10 & 20 \\
\hline 1.4 & 0.332 & 0.15 & 20 & 27 & 30 & 19000 & 23000 & 30500 \\
\hline 1.0 & 0.346 & 0.15 & 45 & 60 & 80 & 37000 & 48000 & 66000 \\
\hline 0.7 & 0.333 & 0.15 & 80 & 98 & 120 & 41000 & 56000 & 74500 \\
\hline
\end{tabular}

TABLE VI. Yield stress and elastic modulus experimentally measured for different silica bead sizes and similar formulations. 
between silica surfaces have been found in similar systems [43]. Besides, the work of adhesion, $W_{S L}$, has not been experimentally measured yet and DLVO theory needs to be included in this approach since experimental results clearly depend on the ionic strength of the suspending fluid.

\section{Experimental identification of the particle size scaling}

In the absence of a universally accepted multiscale model for the overall rheological properties of silica bead suspensions, we check if a particle size scaling exists for our experimental data. We fit the experimental data using power laws

$$
\tau_{c}=A_{n_{\tau}} d_{p}^{n_{\tau}} \quad G=A_{n_{G}} d_{p}^{n_{G}}
$$

where the exponents $n_{\tau}$ and $n_{G}$ are simple numbers assumed to be the same for all the silica suspensions while the coefficients $A_{n_{\tau}}$ and $A_{n_{G}}$ depend on the formulation parameters. Best fits are obtained for $n_{\tau}=-1.94$ and $n_{G}=-1.22$.

Experimental results unambiguously show that the elastic modulus of silica suspensions depend on the particle size in contrast to the DLVO model. Specifically, experimental data for the yield stress are well fitted by a power law with an exponent equal to -1.94 when plotted against the solid volume fraction while DLVO theory predicts an exponent equal to -1 . The models based only on DLVO potentials are consequently not suitable to accurately describe the behavior of silica suspensions.

Even if the model of Pantina and Furst [59] accounts for a size effect on both the elastic modulus and the yield stress, it failed to accurately predict the exponents we calculated from experimental data. The discrepancy is larger for the yield stress exponent $(-1.94 \mathrm{vs}-1.67)$ than for the elastic modulus exponent $(-1.22$ vs -1.33$)$. It might mean that the contact model is more relevant for bending stiffness of interparticle bonds than for local rearrangements of the particles. Anyway, it is not obvious that contact theory accurately describes interparticle interaction between silica particles even if it accounts for tangential forces that are not taken into account by DLVO theory as said in Sec. IV B.

We plot in Fig. 20(a) the elastic modulus scaled by the particle size to the power $n_{G}=-1.22$ against the yield stress scaled by the particle size to the power $n_{\tau}=-1.94$. All the experimental data, obtained for all the formulations and different particle sizes, collapse on a master curve. We use the same scaling to plot the quartz suspension experimental data in Fig. 20(b). Again, all the data fall on a single curve even if the results exhibit a broader dispersion with respect to the monodisperse suspension of spheres. Such distribution doubtlessly comes from the particles polydispersity.

The existence of these master curves reveals that the two coefficients $A_{n_{\tau}}$ and $A_{G}$ are linked by the same relationship whatever the solid volume fraction, the particle size, the ionic strength, or the resting time. As both yield stress and elastic modulus are measured for the same microstructure of the colloidal suspension, it is believed that the master curves observed in Fig. 20 are the microstructure "scalar signature." In addition, existence of master curves suggests that the scaled elastic modulus can play the role of a structural parameter characterizing the instantaneous reorganization of the colloidal suspension. Of course, scaled yield stress can play the same role, but as elastic modulus are easier to measure than the yield stress, experimental validation of a model using the scaled elastic modulus as the structural parameter would be easier.

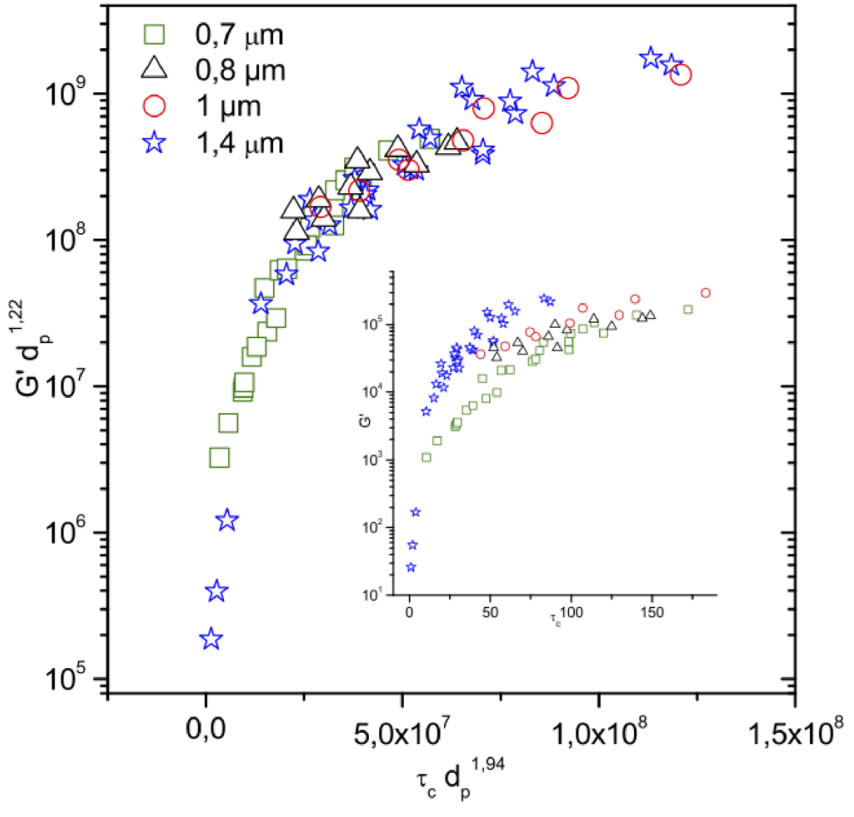

(a) Silica beads

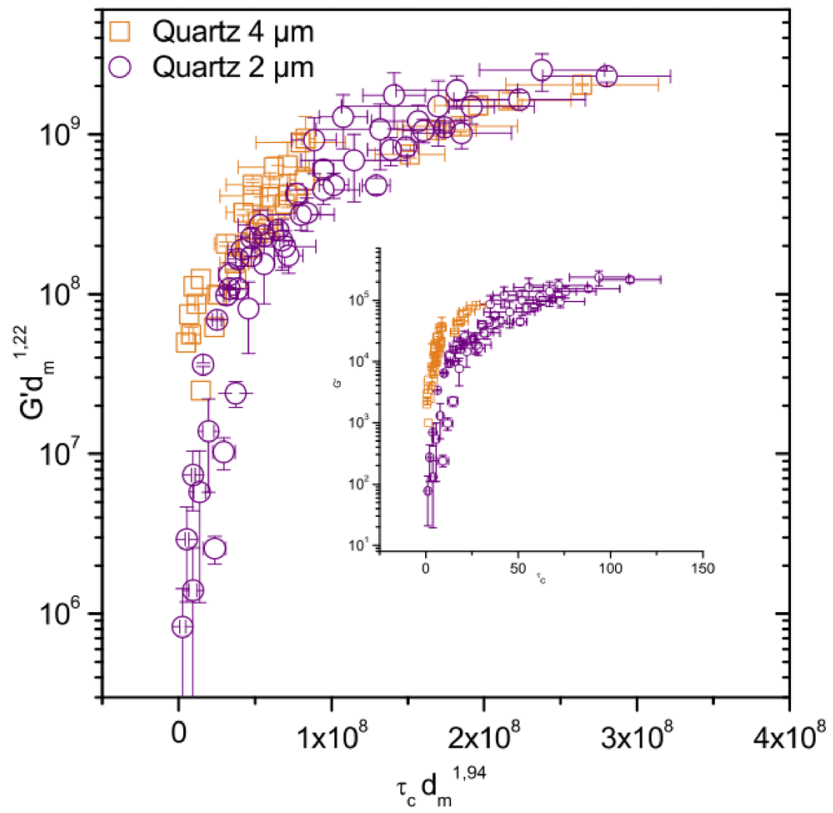

(b) Quartz

FIG. 20. Elastic modulus scaled by the particle size to the power $n_{G}=-1.22$ as a function of yield stress scaled by the particle size to the power $n_{\tau}=-1.94$ for silica bead suspensions (a) and quartz suspensions (b). The insets show the original experimental data. 


\section{CONCLUSION}

We have experimentally studied the elastic modulus and yield stress of suspensions of monodisperse spherical silica particles in an aqueous solution as a function of the particle size, solid volume fraction, ionic strength, and resting time. When plotted in an elastic modulus versus yield stress diagram, all the experimental data for suspensions with same particle size collapse onto a single curve whatever the solid volume fraction, ionic strength, and resting time. This result remains true for angular particles. When plotted against particle size, the experimental data fit a power law well with exponents of $n_{\tau}=-1.94$ for the yield stress and $n_{G}=-1.22$ for the elastic modulus.

In a second step, we plot in a $\tau_{c} d_{p}^{1.94}-G d_{p}^{1.22}$ diagram all the experimental data we collected for monodisperse suspensions of spherical particles with particle diameters of $\{0.7,0.8$, $1.0,1.4\} \mu \mathrm{m}$, solid volume fractions between $33 \%$ and $41 \%$, ionic strength of $\{0.05,0.1,0.15,0.2\}$, and resting time of $\{5$, $10,20\} \mathrm{min}$. All the data fall on a master curve, which is seen as a scalar signature of the colloidal particles microstructure. To check that the power laws and the master curves we identify with monodisperse spherical particles apply to a less controlled system, we also experimentally study suspensions of angular shaped particles having a broader size distribution (crushed silica particles). We observe that experimental data for this system collapse on a master curve in the same $\tau_{c} d_{p}^{1.94}-G d_{p}^{1.22}$ diagram which means that the scaled variables we use are robust enough to account for the size effect of a "real" suspension.

We show that classical models from the literature based on the DLVO theory fail to account for the relationship experimentally observed between the particle size and overall rheological properties. This is likely due to the fact that such models do not take into account the existence of tangential forces or torques at the contact between particles. Size effects are better accounted for by models based on JKR theory, even if the agreement between our experimental data and these models is not perfect since they predict a theoretical exponent of $-5 / 3$ for particle size-yield stress power law and $-4 / 3$ for particle size-elastic modulus.

Confocal microscopy observations performed on silica bead suspensions reveal that the microstructure does not evolve when the suspension is at rest, which is surprising since the overall rheological properties increase strongly with time. This means that the evolution of the yield stress and elastic modulus must be ascribed to small motions of the particles that cannot be observed with our microscope or to strengthening of the interparticle force and contact law due to physicochemical phenomena such as chemical bonds between silica surfaces. These observations contradict classical theory based on Brownian motion and DLVO interparticle forces to explain thixotropy of silica suspensions.

To go further in the understanding of the behavior of such colloidal suspensions, experimental characterization of the interactions between silica particles with a diameter on the order of $1 \mu \mathrm{m}$ suspended in aqueous solution is needed however difficult this seems. Furthermore, a multiscale theory of colloidal suspensions able to account for both DLVO and tangential forces remains to be completed.

\section{ACKNOWLEDGMENT}

Financial support from LafargeHolcim is acknowledged.

\section{References}

[1] Neville, A. M., Properties of Concrete (Prentice Hall, Pearson, 1995).

[2] Russel, W. B., D. A. Saville, and W. R. Schowalter, Colloidal Dispersions (Cambridge University, Harlow, 1989).

[3] Larson, R. G., The Structure and Rheology of Complex Fluids (Oxford University, New York, 1999), Vol. 150.

[4] Coussot, P., Rheometry of Pastes, Suspensions, and Granular Materials: Applications in Industry and Environment (Wiley, Hoboken, NJ, 2005).

[5] Mewis, J., and N. J. Wagner, Colloidal Suspension Rheology (Cambridge University, Cambridge, 2012).

[6] Israelachvili, J. N., Intermolecular and Surface Forces, revised 3rd ed. (Academic, Amsterdam, 2011).

[7] Hoffman, R. L., "Factors affecting the viscosity of unimodal and multimodal colloidal dispersions," J. Rheol. 36(5), 947-965 (1992).

[8] Fagan, M. E., and C. F. Zukoski, "The rheology of charge stabilized silica suspensions," J. Rheol. 41(2), 373-397 (1997).

[9] Zhou, Z., M. J. Solomon, P. J. Scales, and D. V. Boger, "The yield stress of concentrated flocculated suspensions of size distributed particles," J. Rheol. 43(3), 651-671 (1999).

[10] Flatt, R. J., and P. Bowen, "Yodel: A yield stress model for suspensions,” J. Am. Ceram. Soc. 89(4), 1244-1256 (2006).

[11] Eriksson, R., H. Pajari, and J. B. Rosenholm, "Shear modulus of colloidal suspensions: Comparing experiments with theory," J. Colloid Interface Sci. 332(1), 104-112 (2009).

[12] Franks, G. V., S. B. Johnson, P. J. Scales, D. V. Boger, and T. W. Healy, "Ion-specific strength of attractive particle networks," Langmuir 15(13), 4411-4420 (1999).

[13] Teh, E.-J., Y. K. Leong, Y. Liu, B. C. Ong, C. C. Berndt, and S. B. Chen, "Yield stress and zeta potential of washed and highly spherical oxide dispersions-critical zeta potential and hamaker constant," Powder Technol. 198(1), 114-119 (2010).

[14] Zhou, Z., P. J. Scales, and D. V. Boger, "Chemical and physical control of the rheology of concentrated metal oxide suspensions," Chem. Eng. Sci. 56(9), 2901-2920 (2001).

[15] Buscall, R., P. D. A. Mills, J. W. Goodwin, and D. W. Lawson, "Scaling behaviour of the rheology of aggregate networks formed from colloidal particles," J. Chem. Soc., Faraday Trans. 84(12), 4249-4260 (1988).

[16] Lee, J.-D., J.-H. So, and S.-M. Yang, "Rheological behavior and stability of concentrated silica suspensions," J. Rheol. 43(5), 1117-1140 (1999).

[17] Heiney, P. A., R. J. Butera, J. D. Londono, R. V. Davidson, and S. Mazur, "Network growth in the flocculation of concentrated colloidal silica dispersions," J. Phys. Chem. B 104(37), 8807-8821 (2000).

[18] Franks, G. V., "Zeta potentials and yield stresses of silica suspensions in concentrated monovalent electrolytes: Isoelectric point shift and additional attraction,” J. Colloid Interface Sci. 249(1), 44-51 (2002).

[19] Savarmand, S., P.-J. Carreau, F. Bertrand, D. J.-E. Vidal, and M. Moan, "Rheological properties of concentrated aqueous silica suspensions: Effects of pH and ions content," J. Rheol. 47(5), 1133-1149 (2003)

[20] Chen, S., G. Øye, and J. Sjöblom, "Rheological properties of aqueous silica particle suspensions," J. Dispersion Sci. Technol. 26(4), 495-501 (2005).

[21] Amiri, A., G. Oye, and J. Sjöblom, "Influence of pH, high salinity and particle concentration on stability and rheological properties of 
aqueous suspensions of fumed silica," Colloids Surf., A 349(1), 43-54 (2009).

[22] Jiang, T., and C. F. Zukoski, "Dynamic localization and shear-induced hopping of particles: A way to understand the rheology of dense colloidal dispersions," J. Rheol. 58(5), 1277-1299 (2014).

[23] Channell, G. M., and C. F. Zukoski, "Shear and compressive rheology of aggregated alumina suspensions," AIChE J. 43(7), 1700-1708 (1997).

[24] Chen, S., G. Øye, and J. Sjöblom, "Effect of pH and salt on rheological properties of aerosil suspensions," J. Dispersion Sci. Technol. 28(6), 845-853 (2007).

[25] Leong, Y.-K., P. J. Scales, T. W. Healy, and D. V. Boger, "Effect of particle size on colloidal zirconia rheology at the isoelectric point," J. Am. Ceram. Soc. 78(8), 2209-2212 (1995).

[26] Kapur, P. C., P. J. Scales, D. V. Boger, and T. W. Healy, "Yield stress of suspensions loaded with size distributed particles," AIChE J. 43(5), 1171-1179 (1997).

[27] Scales, P. J., S. B. Johnson, T. W. Healy, and P. C. Kapur, "Shear yield stress of partially flocculated colloidal suspensions," AIChE J. 44(3), 538-544 (1998).

[28] Potanin, A. A., R. De Rooij, D. Van den Ende, and J. Mellema, "Microrheological modeling of weakly aggregated dispersions," J. Chem. Phys. 102(14), 5845-5853 (1995).

[29] Weitz, D. A., and M. Oliveria, "Fractal structures formed by kinetic aggregation of aqueous gold colloids," Phys. Rev. Lett. 52(16), 1433-1436 (1984).

[30] Mari, R., R. Seto, J. F. Morris, and M. M. Denn, "Shear thickening, frictionless and frictional rheeologies in non-Brownian suspensions," J. Rheol. 58, 1963-1724 (2014).

[31] Seto, R., R. Botet, G. K. Auernhammer, and H. Briesen, "Restructuring of colloidal aggregates in shear flow: Coupling interparticle contact models with Stokesian dynamics," Eur. Phys. J. E 35(128) (2012).

[32] Seto, R., R. Botet, and H. Briesen, "Viscosity of rigid and breakable aggregate suspensions-Stokesian dynamics for rigid aggregates," in Progress in Colloid and Polymer Science, edited by V. Starov and P. Griffiths (Springer, Berlin, Heidelberg, 2012), Vol. 129, pp. 85-90.

[33] Seto, R., R. Botet, G. K. Meireles, M. Auernhammer, and B. Cabane, "Compressive consolidation of strongly aggregated particle gels," J. Rheol. 27(5), 1347-1366 (2013).

[34] Derjaguin, B. V., and L. Landau, "The theory of stability of highly charged lyophobic sols and coalescence of highly charged particles in electrolyte solutions," Acta Physicochim. URSS 14, 633-652 (1941).

[35] Verwey, E. J. W., and J. Th. G. Overbeek, Theory of Lyophobic Colloids (Elsevier, Amsterdam, 1948).

[36] Hamaker, H. C., "The London-van der Waals attraction between spherical particles," Physica 4(10), 1058-1072 (1937).

[37] Stöber, W., A. Fink, and E. Bohn, "Controlled growth of monodisperse silica spheres in the micron size range,” J. Colloid Interface Sci. 26(1), 62-69 (1968).

[38] Bogush, G. H., M. A. Tracy, and C. F. Zukoski, "Preparation of monodisperse silica particles: Control of size and mass fraction," J. NonCryst. Solids 104(1), 95-106 (1988)

[39] Lindberg, R., G. Sundholm, B. Pettersen, J. Sjoblom, and S. E. Friberg, "Multivariate analysis of the size dependence of monodisperse silica particles prepared according to the sol-gel technique," Colloids Surf., A 123, 549-560 (1997).
[40] Ibrahim, I. A. M., A. A. F. Zikry, and M. A. Sharaf, "Preparation of spherical silica nanoparticles: Stöber silica," J. Am. Sci. 6(11), 985-989 (2010).

[41] Nozawa, K., H. Gailhanou, L. Raison, P. Panizza, H. Ushiki, E. Sellier, J. P. Delville, and M. H. Delville, "Smart control of monodisperse stöber silica particles: Effect of reactant addition rate on growth process," Langmuir 21(4), 1516-1523 (2005).

[42] Kang, S., S. I. Hong, C. R. Choe, M. Park, S. Rim, and J. Kim, "Preparation and characterization of epoxy composites filled with functionalized nanosilica particles obtained via sol-gel process," Polymer 42(3), 879-887 (2001).

[43] Iler, R. K., The Chemistry of Silica: Solubility, Polymerization, Colloid and Surface Properties, and Biochemistry (Wiley, New York; Chichester; Brisbane; Toronto, 1979).

[44] Smoluchowski, M. V., Handbook of Electricity and Magnetism (Barth, Leipzig, 1921).

[45] Johnson, S. B., A. S. Russell, and P. J. Scales, "Volume fraction effects in shear rheology and electroacoustic studies of concentrated alumina and kaolin suspensions," Colloids Surf., A 141(1), 119-130 (1998).

[46] Callaghan, P. T., Principles of Nuclear Magnetic Resonance Spectroscopy (Clarendon, Oxford, 1991).

[47] Ovarlez, G., F. Bertrand, and S. Rodts, "Local determination of the constitutive law of a dense suspension of noncolloidal particles through magnetic resonance imaging," J. Rheol. 50(3), 259-292 (2006).

[48] Ovarlez, G., and X. Chateau, "Influence of shear stress applied during flow stoppage and rest period on the mechanical properties of thixotropic suspensions," Phys. Rev. E 77(6), 061403 (2008).

[49] Trompette, J.-L., and M. J. Clifton, "Influence of ionic specificity on the microstructure and the strength of gelled colloidal silica suspensions," J. Colloid Interface Sci. 276(2), 475-482 (2004).

[50] Zhou, Z., Rheology of metal oxide suspensions, Ph.D. thesis, University of Melbourne, 2000.

[51] Smoluchowski, M. V., "Outline of the coagulation kinetics of colloidal solutions,” Kolloid-Z. 21(3), 98-104 (1917).

[52] Horn, R. G., D. T. Smith, and W. Haller, "Surface forces and viscosity of water measured between silica sheets," Chem. Phys. Lett. 162(4-5), 404-408 (1989).

[53] Pashley, R. M., and J. N. Israelachvili, "Dlvo and hydration forces between mica surfaces in $\mathrm{Mg}^{2+}, \mathrm{Ca}^{2+}, \mathrm{Sr}^{2+}$, and $\mathrm{Ba}^{2+}$ chloride solutions," J. Colloid Interface Sci. 97(2), 446-455 (1984).

[54] Oosawa, F., Polyelectrolytes (Marcel D., New York, 1971).

[55] Jennsson, B., H. Wennerstroem, and B. Halle, "Ion distributions in lamellar liquid crystals. A comparison between results from Monte Carlo simulations and solutions of the Poisson-Boltzmann equation," J. Phys. Chem. A 84(17), 2179-2185 (1980).

[56] Kjellander, R., S. Marcelja, R. M. Pashley, and J. P. Quirk, "Doublelayer ion correlation forces restrict calcium-clay swelling," J. Phys. Chem. 92(23), 6489-6492 (1988).

[57] Pantina, J. P., and E. M. Furst, "Elasticity and critical bending moment of model colloidal aggregates," Phys. Rev. Lett. 94(13), 138301 (2005).

[58] Johnson, K. L., K. Kendall, and A. D. Roberts, "Surface energy and the contact of elastic solids," Proc. R. Soc. A 324(1558), 301-313 (1971).

[59] Pantina, J. P., and E. M. Furst, "Directed assembly and rupture mechanics of colloidal aggregates," Langmuir 20(10), 3940-3946 (2004). 